\title{
Uso de la ciencia de datos y el aprendizaje automático para analizar la aplicación GeoGebra en el proceso educativo
}

\author{
Ricardo-Adán Salas-Rueda \\ ricardo.salas@icat.unam.mx \\ Instituto de ciencias aplicadas y Tecnología, Universidad Nacional Autónoma de México, México.
}

\author{
Rodrigo-David Salas-Rueda \\ rodrigodavidsalas@hotmail.com
}

Universidad Autónoma Metropolitana, México.

\begin{abstract}
Resumen
Esta investigación mixta analiza el uso de la aplicación GeoGebra en el proceso de enseñanza-aprendizaje sobre la Probabilidad Binomial por medio de la ciencia de datos y el aprendizaje automático. La muestra está compuesta por 61 alumnos de la asignatura Instrumentación estadística para los negocios. Este estudio analiza el impacto de los contenidos, la estética y la simulación de la aplicación GeoGebra en el proceso educativo por medio del aprendizaje automático (regresión lineal) con $60 \%, 70 \%$ y $80 \%$ de entrenamiento. Además, la herramienta Rapidminer permite identificar los modelos predictivos sobre el uso de la aplicación GeoGebra en el contexto educativo por medio de la técnica árbol de decisión. Por otro lado, la herramienta Maxqda permite realizar el análisis cualitativo a través de la nube de palabras. Los resultados indican que la aplicación GeoGebra facilita el proceso de enseñanza-aprendizaje sobre la Probabilidad Binomial, desarrolla las habilidades matemáticas, mejora la asimilación del conocimiento y propicia el rol activo del estudiante. Por último, los docentes pueden innovar las actividades escolares sobre la estadística y transformar el comportamiento de los alumnos durante el aprendizaje por medio de la aplicación GeoGebra.
\end{abstract}

\section{Palabras clave}

GeoGebra; enseñanza superior; ciencia de datos; aprendizaje automático; tecnología 


\title{
Use of data science and machine learning to analyze GeoGebra application in the educational process
}

\author{
Ricardo-Adán Salas-Rueda \\ ricardo.salas@icat.unam.mx \\ Universidad Nacional Autónoma de México, México. \\ Rodrigo-David Salas-Rueda \\ rodrigodavidsalas@hotmail.com \\ Universidad Autónoma Metropolitana, México.
}

\begin{abstract}
This mixed research analyzes the use of GeoGebra application in the teaching-learning process on Binomial Probability through data science and machine learning. The sample consists of 61 students of the Statistical Instrumentation for Business course. This study analyzes the impact of content, aesthetics and simulation of GeoGebra application in the educational process through machine learning (linear regression) with 60\%, 70\% and $80 \%$ of training. In addition, Rapidminer tool allows identifying predictive models about the use of GeoGebra application in the educational context by means of the decision tree technique. On the other hand, Maxqda tool allows qualitative analysis through the word cloud. The results indicate that GeoGebra application facilitates the teaching-learning process on Binomial Probability, develops the mathematical skills, improves the assimilation of knowledge and promotes the active role of the student. Finally, teachers can innovate school activities on statistics and transform the behavior of students in learning through GeoGebra application.
\end{abstract}

\section{Keywords}

GeoGebra; higher education; data science; machine learning; technology 


\section{Introducción}

La Sociedad del Siglo XXI demanda desarrollar y perfeccionar las habilidades digitales de los docentes relacionadas con la búsqueda, la evaluación, el uso, la modificación y la creación de prácticas educativas por medio de la tecnología (Mavroudi y Tsagari, 2018). De hecho, los profesores pueden actualizar las actividades escolares y crear nuevos espacios virtuales a través de las herramientas digitales, las aplicaciones web y el software educativo (Landa, Martínez, López y Ramírez, 2018; Porat, Blau y Barak, 2018).

La integración exitosa de las aplicaciones tecnológicas en el campo educativo está relacionada con el cumplimiento eficaz de los objetivos planteados en el proceso de enseñanza-aprendizaje (Banerjee y Murthy, 2018; Hu, Gong, Lai y Leung, 2018; Rooij y Zirkle, 2016). Además, el empleo de la tecnología dentro y/o fuera del salón de clases permite incrementar la satisfacción y motivación de los estudiantes (Salas, Vázquez y Lugo, 2016; Weidlich y Bastiaens, 2018).

Diversos autores (p.ej., Hobbs y Tuzel, 2017; Landa, Martínez, López y Ramírez, 2018; Scott, Nerminathan, Alexander, Phelps y Harrison, 2017) afirman que es necesario incrementar el uso de las aplicaciones en el campo educativo con el propósito de mejorar las condiciones de enseñanzaaprendizaje. Hoy en día, Internet es un medio idóneo para identificar y seleccionar los programas, las aplicaciones web, las herramientas digitales y los recursos didácticos necesarios para cubrir las expectativas y necesidades de los estudiantes (Landa, Martínez, López y Ramírez, 2018; Salas y Vázquez, 2017).

Las universidades mexicanas tienen el reto de innovar el proceso de enseñanza-aprendizaje en el Siglo XXI. En particular, los alumnos de las Licenciaturas en Administración, Comercio, Contaduría, Informática y Mercadotecnia tienen dificultades para asimilar el conocimiento y desarrollar las habilidades matemáticas sobre los temas de la Probabilidad Binomial en la asignatura Instrumentación estadística para los negocios.

Una de las alternativas para actualizar las actividades escolares dentro y fuera del salón de clases es el uso de la tecnología. Por ejemplo, GeoGebra es una aplicación web gratuita que permite innovar el proceso educativo relacionado con las matemáticas (Granberg y Olsson, 2015; Salas, 2018; Takaci, Stankov y Milanovic, 2015).

Esta investigación mixta tiene como objetivo analizar el impacto de la aplicación GeoGebra en el proceso de enseñanza-aprendizaje sobre la Probabilidad Binomial por medio de la ciencia de datos y el aprendizaje automático (regresión lineal).

Por lo tanto, las preguntas de investigación son:

- ¿Cuál es el impacto de la aplicación GeoGebra en el proceso de enseñanza-aprendizaje sobre la Probabilidad Binomial durante la asimilación del conocimiento, el desarrollo de las habilidades matemáticas y el rol activo del estudiante considerando el aprendizaje automático (regresión lineal)?

- ¿Cuáles son los modelos predictivos sobre el uso de la aplicación GeoGebra en el proceso de enseñanza-aprendizaje sobre la Probabilidad Binomial (técnica árbol de decisión)?

- ¿Cuáles son las percepciones de los alumnos sobre el uso de la aplicación GeoGebra en el proceso de enseñanza-aprendizaje sobre la Probabilidad Binomial? 


\section{Tecnología en el campo educativo}

Los avances sobre las Tecnologías de la Información y Comunicación (TIC) están propiciando que las instituciones educativas organicen y construyan nuevos entornos virtuales para el aprendizaje y la enseñanza (Hernández, Acosta, Gordillo y Sandoval, 2018; Salas y Salas, 2018). De hecho, la educación a distancia (e-learning) es una forma de enseñanza en Internet que permite la creación de ambientes interactivos, eficientes y accesibles para el aprendizaje (Hernández, Acosta, Gordillo y Sandoval, 2018). Durante el Siglo XXI, los docentes están utilizando diversas aplicaciones web (p.ej., GeoGebra) bajo la modalidad e-learning con la finalidad de desarrollar las competencias en los estudiantes (Salas, 2018).

Hoy en día, las actividades de la modalidad a distancia permiten a los estudiantes administrar el ritmo de aprendizaje en cualquier momento y lugar (Alkis y Taskaya, 2018; Dolan, Hancock y Wareing, 2015). En particular, la modalidad Blended learning combina las actividades escolares desarrolladas en el salón de clases (sesiones presenciales) con las tareas realizadas en la modalidad a distancia (Alkis y Taskaya, 2018).

Las TIC están transformando las funciones de los docentes y alumnos durante el proceso de enseñanza-aprendizaje sobre las matemáticas en los niveles educativos de primaria (Khalil, Farooq, Cakıroglu, Khalil y Khan, 2018), secundaria (Zulnaidi y Zamri, 2017), preparatoria (Bhagat y Chang, 2015) y universidad (Salas, 2018). Incluso, las herramientas digitales y aplicaciones web incrementan la motivación de los estudiantes durante el aprendizaje, mejoran la distribución de la información en la red y facilitan la participación activa de los alumnos (Landa, Martínez, López y Ramírez, 2018; Yang, Kuo, Ji y McTigue, 2018).

En el campo de las ciencias se han diseñado e implementando diversos sistemas web como STACK (System for Teaching and Assessment using a Computer algebra Kernel) para fomentar el desarrollo de las habilidades matemáticas en los estudiantes (Hernández, Acosta, Gordillo y Sandoval, 2018). Del mismo modo, las aplicaciones web (p.ej., GeoGebra) son utilizadas en el campo de las matemáticas para mejorar las condiciones de enseñanza-aprendizaje y crear nuevas experiencias educativas (Salas, 2018; Wassie y Zergaw, 2019).

\section{a. Uso de la aplicación GeoGebra en el campo educativo}

Diversos autores (p.ej., Granberg y Olsson, 2015; Salas, 2018; Takaci, Stankov y Milanovic, 2015) proponen el uso de la aplicación GeoGebra en las actividades escolares con la finalidad de mejorar el proceso de enseñanza-aprendizaje sobre las matemáticas. Por ejemplo, la aplicación GeoGebra facilita el desarrollo de las competencias en los estudiantes y la asimilación del conocimiento sobre los temas de cálculo (Takaci, Stankov y Milanovic, 2015), desigualdades lineales (Salas, 2018), geometría (Bhagat y Chang, 2015) y funciones (Granberg y Olsson, 2015; Zulnaidi y Zamri, 2017). Por ejemplo, Salas (2018) utilizó la aplicación GeoGebra en la asignatura Matemáticas Intermedia para los negocios con el propósito de facilitar la asimilación del conocimiento sobre las desigualdades lineales y mejorar el desarrollo de las habilidades matemáticas.

Cabe mencionar que la interfaz de la aplicación GeoGebra permite el razonamiento matemático por medio de una retroalimentación gráfica, rápida, sencilla, agradable y creativa (Granberg y Olsson, 2015). Incluso, la aplicación GeoGebra incrementa el interés de los estudiantes durante el proceso educativo, mejora el rendimiento académico y facilita la construcción de espacios virtuales educativos (Wassie y Zergaw, 2019).

En el nivel primaria, la incorporación de la aplicación GeoGebra mejoró las condiciones de enseñanza-aprendizaje (Khalil, Farooq, Cakıroglu, Khalil y Khan, 2018). Zulnaidi y Zamri (2017) señalan que la aplicación GeoGebra facilitó el proceso educativo sobre los temas de las funciones en el nivel secundaria. Del mismo modo, Bhagat y Chang (2015) utilizaron la aplicación GeoGebra 
para facilitar la asimilación del conocimiento sobre la geometría en el nivel de preparatoria.

Por último, las TIC están modificando la planeación y organización de las actividades escolares en el campo de las matemáticas (Granberg y Olsson, 2015; Wassie y Zergaw, 2019). En particular, GeoGebra es una aplicación web gratuita y accesible en Internet que facilita la creación de nuevas experiencias educativas en el campo de las matemáticas (Granberg y Olsson, 2015; Salas, 2018; Takaci, Stankov y Milanovic, 2015; Zulnaidi y Zamri, 2017).

\section{Metodología}

El objetivo de esta investigación mixta es analizar el impacto de la aplicación GeoGebra en el proceso de enseñanza-aprendizaje sobre la Probabilidad Binomial por medio de la ciencia de datos y el aprendizaje automático.

Este estudió utilizó la herramienta Rapidminer para construir los modelos predictivos (técnica árbol de decisión) y calcular el aprendizaje automático con $60 \%, 70 \%$ y $80 \%$ de entrenamiento, el software Maxqda para realizar el análisis cualitativo (nube de palabras) y la hoja de cálculo para obtener la regresión lineal.

\section{a. Participantes}

La muestra de esta investigación está conformada por 61 alumnos (28 hombres y 33 mujeres) de las Licenciaturas en Administración $(n=14,22.95 \%)$, Comercio $(n=12,19.67 \%)$, Contaduría $(n=19,31.15 \%)$, Informática $(n=2,3.28 \%)$ y Mercadotecnia $(n=14,22.95 \%)$, los cuales cursaron la asignatura Instrumentación estadística para los negocios en una universidad privada de la Ciudad de México durante el ciclo escolar 2018. La edad promedio de los estudiantes es 19.01 años.

\section{b. Procedimiento}

El procedimiento de esta investigación inició con el uso del modelo ADDIE para planear y organizar el proceso de enseñanza-aprendizaje sobre la Probabilidad Binomial por medio de la aplicación GeoGebra (Ver Tabla 1).

\begin{tabular}{|c|c|c|c|}
\hline No. & Etapa & Elemento & Descripción \\
\hline \multirow{6}{*}{1} & \multirow{6}{*}{ Análisis } & \multirow{3}{*}{ Objetivo } & $\begin{array}{l}\text { Comprender los temas sobre la probabilidad } \\
\text { binomial }\end{array}$ \\
\hline & & & $\begin{array}{l}\text { Analizar las fórmulas sobre la probabilidad } \\
\text { binomial }\end{array}$ \\
\hline & & & $\begin{array}{l}\text { Aplicar los conceptos sobre la probabilidad } \\
\text { binomial en el contexto práctico }\end{array}$ \\
\hline & & Recurso tecnológico & Aplicación GeoGebra \\
\hline & & Asignatura & Instrumentación estadística para los negocios \\
\hline & & Características de los & Los alumnos tienen una edad promedio de \\
\hline
\end{tabular}




\begin{tabular}{|c|c|c|c|}
\hline & & alumnos & $\begin{array}{l}19.01 \text { años y cursan el segundo semestre de } \\
\text { las Licenciaturas en Administración, } \\
\text { Comercio, Contaduría, Informática y } \\
\text { Mercadotecnia }\end{array}$ \\
\hline \multirow{8}{*}{2} & \multirow{8}{*}{ Diseño } & \multirow{4}{*}{ Objetivos particulares } & $\begin{array}{l}\text { Comprender el cálculo de la probabilidad } \\
\text { binomial puntual }\end{array}$ \\
\hline & & & $\begin{array}{l}\text { Comprender el cálculo de la probabilidad } \\
\text { binomial de intervalos }\end{array}$ \\
\hline & & & $\begin{array}{l}\text { Comprender el uso de la probabilidad } \\
\text { binomial puntual en el contexto práctico }\end{array}$ \\
\hline & & & $\begin{array}{l}\text { Comprender el uso de la probabilidad } \\
\text { binomial de intervalos en el contexto práctico }\end{array}$ \\
\hline & & \multirow{4}{*}{$\begin{array}{l}\text { Incorporación de la } \\
\text { aplicación GeoGebra } \\
\text { en las actividades } \\
\text { escolares }\end{array}$} & $\begin{array}{l}\text { Práctica de laboratorio } 1 \text { sobre el cálculo de } \\
\text { la probabilidad binomial puntual, p.ej., } F(1)\end{array}$ \\
\hline & & & $\begin{array}{l}\text { Práctica de laboratorio } 2 \text { sobre el cálculo de } \\
\text { la probabilidad binomial de intervalos, p.ej., } \\
F(x \leq 2)\end{array}$ \\
\hline & & & $\begin{array}{l}\text { Práctica de laboratorio } 3 \text { sobre el uso de la } \\
\text { probabilidad binomial puntual en el contexto } \\
\text { práctico, p.ej., ejercicio sobre la compra de } \\
\text { productos }\end{array}$ \\
\hline & & & $\begin{array}{l}\text { Práctica de laboratorio } 4 \text { sobre el uso de la } \\
\text { probabilidad binomial de intervalos en el } \\
\text { contexto práctico, p.ej., ejercicio sobre la } \\
\text { preferencia del consumidor }\end{array}$ \\
\hline \multirow[b]{2}{*}{3} & \multirow[b]{2}{*}{ Desarrollo } & Plan de trabajo & $\begin{array}{l}\text { Al finalizar cada una de las sesiones } \\
\text { presenciales de la Unidad probabilidad } \\
\text { binomial, el alumno realizará } 1 \text { práctica de } \\
\text { laboratorio }\end{array}$ \\
\hline & & $\begin{array}{l}\text { Distribución del } \\
\text { material }\end{array}$ & $\begin{array}{l}\text { Los alumnos de las Licenciaturas en } \\
\text { Administración, Comercio, Contaduría, } \\
\text { Informática y Mercadotecnia acceden a las } \\
\text { prácticas de laboratorio por medio de un sitio } \\
\text { web }\end{array}$ \\
\hline 4 & Implementación & $\begin{array}{l}\text { Presentación de la } \\
\text { información }\end{array}$ & $\begin{array}{l}\text { La Tabla } 2 \text { muestra algunos ejercicios de la } \\
\text { prácticas de laboratorio }\end{array}$ \\
\hline 5 & Evaluación & Cuestionario & $\begin{array}{l}\text { La Tabla } 3 \text { muestra el instrumento de } \\
\text { medición para evaluar el impacto de la } \\
\text { aplicación GeoGebra en el proceso de } \\
\text { enseñanza-aprendizaje }\end{array}$ \\
\hline
\end{tabular}

Tabla 1. Uso del modelo ADDIE en el proceso educativo

Fuente: Elaboración propia 
La Tabla 2 muestra algunos de los ejercicios realizados en las prácticas de laboratorio sobre el cálculo de la Probabilidad Binomial por medio de la aplicación GeoGebra.

\begin{tabular}{|c|c|c|}
\hline $\begin{array}{l}\text { Práctica de } \\
\text { laboratorio }\end{array}$ & Ejemplo de ejercicios & Aplicación \\
\hline 1 & $\begin{array}{l}\text { Emplea la aplicación GeoGebra para calcular la probabilidad binomial. } \\
\text { Para ingresar a esta herramienta tecnológica utiliza la siguiente } \\
\text { dirección web: https://www.geogebra.org/classic/probability } \\
\text { Calcula las siguientes probabilidades binomiales si } n=7 \text { y } p=0.7: F(0) \text {, } \\
F(1), F(2), F(3), F(4), F(5), F(6) \text { y } F(7)\end{array}$ & GeoGebra \\
\hline 2 & $\begin{array}{l}\text { Emplea la aplicación GeoGebra para calcular la probabilidad binomial. } \\
\text { Para ingresar a esta herramienta tecnológica utiliza la siguiente } \\
\text { dirección web: https://www.geogebra.org/classic/probability } \\
\text { Calcula las siguientes probabilidades binomiales si } n=12 \text { y } p=0.6 \text { : } F(2) \text {, } \\
F(3), F(4), F(5), F(x \leq 2), F(x \leq 3), F(x \geq 3), F(x \geq 4) \text { y } F(x \geq 5)\end{array}$ & GeoGebra \\
\hline 3 & $\begin{array}{l}\text { Emplea la aplicación GeoGebra para calcular la probabilidad binomial. } \\
\text { Para ingresar a esta herramienta tecnológica utiliza la siguiente } \\
\text { dirección web: https://www.geogebra.org/classic/probability } \\
\text { Considere las decisiones de } 4 \text { clientes para comprar un producto. } \\
\text { Utiliza la distribución de probabilidad binomial para encontrar que: } \\
\text { Ningún cliente compre, } 2 \text { clientes compren y } 3 \text { clientes compren. } \\
\text { Con base a la experiencia, el gerente de la tienda estima la } \\
\text { probabilidad de que el cliente haga una compra es de } 0.8 \text {. }\end{array}$ & GeoGebra \\
\hline 4 & $\begin{array}{l}\text { Emplea la aplicación GeoGebra para calcular la probabilidad binomial. } \\
\text { Para ingresar a esta herramienta tecnológica utiliza la siguiente } \\
\text { dirección web: https://www.geogebra.org/classic/probability } \\
\text { Considere las decisiones de } 6 \text { clientes para fumar cigarro. Utiliza la } \\
\text { distribución de probabilidad binomial para encontrar que: Ningún } \\
\text { cliente fuma, Más de } 4 \text { clientes fuman y Menos de } 5 \text { clientes fuman. } \\
\text { La probabilidad para fumar es } 0.4 \text {. }\end{array}$ & GeoGebra \\
\hline
\end{tabular}

Tabla 2. Ejercicios sobre las prácticas de laboratorio

Fuente: Elaboración propia

Esta investigación mixta analiza el impacto de la aplicación GeoGebra (contenidos, estética y simulación) durante el proceso de enseñanza-aprendizaje (asimilación del conocimiento, desarrollo de habilidades y rol activo). La Figura 1 muestra el modelo de aceptación tecnológica sobre el uso de la aplicación GeoGebra en el campo educativo. 


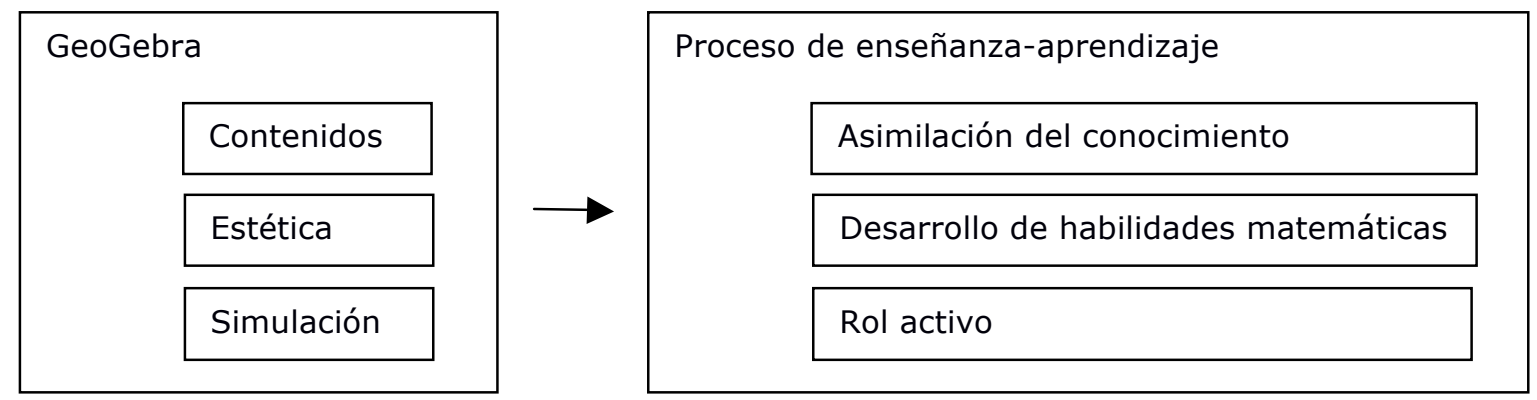

Figura 1. Modelo de aceptación tecnológica

Fuente: Elaboración propia

Las hipótesis de investigación relacionadas con los contenidos de la aplicación GeoGebra y el proceso de enseñanza-aprendizaje son:

- H1: Los contenidos de la aplicación GeoGebra influye positivamente la asimilación del conocimiento sobre la Probabilidad Binomial

- H2: Los contenidos de la aplicación GeoGebra influye positivamente el desarrollo de las habilidades matemáticas sobre la Probabilidad Binomial

- H3: Los contenidos de la aplicación GeoGebra influye positivamente el rol activo del estudiante durante el aprendizaje de la Probabilidad Binomial

Las hipótesis de investigación sobre la estética de la aplicación GeoGebra y el proceso de enseñanza-aprendizaje son:

- H4: La estética de la aplicación GeoGebra influye positivamente la asimilación del conocimiento sobre la Probabilidad Binomial

- H5: La estética de la aplicación GeoGebra influye positivamente el desarrollo de las habilidades matemáticas sobre la Probabilidad Binomial

- H6: La estética de la aplicación GeoGebra influye positivamente el rol activo del estudiante durante el aprendizaje de la Probabilidad Binomial

Las hipótesis de investigación sobre la simulación de la aplicación GeoGebra y el proceso de enseñanza-aprendizaje son:

- H7: La simulación de la aplicación GeoGebra influye positivamente la asimilación del conocimiento sobre la Probabilidad Binomial

- H8: La simulación de la aplicación GeoGebra influye positivamente el desarrollo de las habilidades matemáticas sobre la Probabilidad Binomial

- H9: La simulación de la aplicación GeoGebra influye positivamente el rol activo del estudiante durante el aprendizaje de la Probabilidad Binomial

La herramienta Rapidminer permite identificar los modelos predictivos sobre el uso de la aplicación GeoGebra en el proceso educativo sobre la Probabilidad Binomial por medio de la técnica árbol de decisión. Durante la construcción de los modelos predictivos, esta investigación utiliza la información relacionada con el proceso de enseñanza-aprendizaje (asimilación del conocimiento, desarrollo de habilidades matemáticas y rol activo del estudiante), la aplicación GeoGebra 
(contenidos, estética y simulador) y el perfil de los alumnos (edad, género y licenciatura).

Los modelos predictivos sobre los Contenidos de la aplicación GeoGebra y el proceso educativo son:

- Modelo predictivo 1: Impacto de la aplicación GeoGebra (contenidos) para la asimilación del conocimiento sobre la Probabilidad Binomial

- Modelo predictivo 2: Impacto de la aplicación GeoGebra (contenidos) para el desarrollo de las habilidades matemáticas sobre la Probabilidad Binomial

- Modelo predictivo 3: Impacto de la aplicación GeoGebra (contenidos) para el rol activo del estudiante

Los modelos predictivos sobre la Estética de la aplicación GeoGebra y el proceso educativo sobre la Probabilidad Binomial son:

- Modelo predictivo 4: Impacto de la aplicación GeoGebra (estética) para la asimilación del conocimiento sobre la Probabilidad Binomial

- Modelo predictivo 5: Impacto de la aplicación GeoGebra (estética) para el desarrollo de las habilidades matemáticas sobre la Probabilidad Binomial

- Modelo predictivo 6: Impacto de la aplicación GeoGebra (estética) para el rol activo del estudiante

Asimismo, los modelos predictivos sobre la Simulación de la aplicación GeoGebra y el proceso educativo son:

- Modelo predictivo 7: Impacto de la aplicación GeoGebra (simulación) para la asimilación del conocimiento sobre la Probabilidad Binomial

- Modelo predictivo 8: Impacto de la aplicación GeoGebra (simulación) para el desarrollo de las habilidades matemáticas sobre la Probabilidad Binomial

- Modelo predictivo 9: Impacto de la aplicación GeoGebra (simulación) para el rol activo del estudiante

\section{c. Recolección de datos}

La Tabla 3 muestra el instrumento de medición utilizado en esta investigación para analizar el impacto del impacto de la aplicación GeoGebra en el proceso de enseñanza-aprendizaje sobre la Probabilidad Binomial por medio de la ciencia de datos y el aprendizaje automático.

\begin{tabular}{|c|c|c|c|c|c|c|}
\hline No. & Variable & Dimensión & Pregunta & Respuesta & $\mathrm{n}$ & $\%$ \\
\hline \multirow{6}{*}{1} & \multirow{6}{*}{$\begin{array}{c}\text { Perfil del } \\
\text { estudiante }\end{array}$} & \multirow{3}{*}{ Género } & \multirow[t]{3}{*}{ 1. Indica tu género } & & & \\
\hline & & & & Hombre & 28 & $45.90 \%$ \\
\hline & & & & Mujer & 33 & $54.10 \%$ \\
\hline & & \multirow{3}{*}{ Edad } & \multirow[t]{3}{*}{ 2. Indica tu edad } & & & \\
\hline & & & & 18 años & 20 & $32.79 \%$ \\
\hline & & & & 19 años & 25 & $40.98 \%$ \\
\hline
\end{tabular}




\begin{tabular}{|c|c|c|c|c|c|c|}
\hline & & & & 20 años & 12 & $19.67 \%$ \\
\hline & & & & 21 años & 3 & $4.92 \%$ \\
\hline & & & & 22 años & 1 & $1.64 \%$ \\
\hline & & & & 23 años & 0 & $0.00 \%$ \\
\hline & & & & 24 años & 0 & $0.00 \%$ \\
\hline & & \multirow{6}{*}{ Licenciatura } & \multirow{6}{*}{$\begin{array}{l}\text { 3. Indica tu } \\
\text { licenciatura }\end{array}$} & & & \\
\hline & & & & Administración & 14 & $22.95 \%$ \\
\hline & & & & Contaduría & 12 & $19.67 \%$ \\
\hline & & & & Comercio & 19 & $31.15 \%$ \\
\hline & & & & Informática & 2 & $3.28 \%$ \\
\hline & & & & Mercadotecnia & 14 & $22.95 \%$ \\
\hline \multirow{18}{*}{2} & \multirow{18}{*}{$\begin{array}{l}\text { Aplicación } \\
\text { GeoGebra }\end{array}$} & \multirow{6}{*}{ Contenidos } & \multirow{6}{*}{$\begin{array}{l}\text { 4. Los contenidos de } \\
\text { GeoGebra facilitan el } \\
\text { proceso de } \\
\text { enseñanza- } \\
\text { aprendizaje }\end{array}$} & & & \\
\hline & & & & Totalmente (1) & 40 & $65.57 \%$ \\
\hline & & & & Bastante (2) & 21 & $34.43 \%$ \\
\hline & & & & Regular (3) & 0 & $0.00 \%$ \\
\hline & & & & Poco (4) & 0 & $0.00 \%$ \\
\hline & & & & Nada (5) & 0 & $0.00 \%$ \\
\hline & & \multirow{6}{*}{ Estética } & \multirow{6}{*}{$\begin{array}{l}\text { 5. La estética de } \\
\text { GeoGebra facilita el } \\
\text { proceso de } \\
\text { enseñanza- } \\
\text { aprendizaje }\end{array}$} & & & \\
\hline & & & & Totalmente (1) & 40 & $65.57 \%$ \\
\hline & & & & Bastante (2) & 16 & $26.23 \%$ \\
\hline & & & & Regular (3) & 5 & $8.20 \%$ \\
\hline & & & & Poco (4) & 0 & $0.00 \%$ \\
\hline & & & & Nada (5) & 0 & $0.00 \%$ \\
\hline & & \multirow{6}{*}{ Simulación } & \multirow{6}{*}{$\begin{array}{l}\text { 6. La simulación de } \\
\text { GeoGebra facilita el } \\
\text { proceso de } \\
\text { enseñanza- } \\
\text { aprendizaje }\end{array}$} & & & \\
\hline & & & & Totalmente (1) & 41 & $67.21 \%$ \\
\hline & & & & Bastante (2) & 18 & $29.51 \%$ \\
\hline & & & & Regular (3) & 2 & $3.28 \%$ \\
\hline & & & & Poco (4) & 0 & $0.00 \%$ \\
\hline & & & & Nada (5) & 0 & $0.00 \%$ \\
\hline \multirow{2}{*}{3} & \multirow{2}{*}{$\begin{array}{l}\text { Proceso } \\
\text { educativo } \\
\text { sobre la }\end{array}$} & \multirow{2}{*}{$\begin{array}{l}\text { Asimilación } \\
\text { del }\end{array}$} & \multirow{2}{*}{$\begin{array}{l}\text { 7. La aplicación } \\
\text { GeoGebra facilita la } \\
\text { asimilación de }\end{array}$} & & & \\
\hline & & & & Totalmente (1) & 35 & $57.38 \%$ \\
\hline
\end{tabular}




\begin{tabular}{|c|c|c|c|c|c|}
\hline \multirow{16}{*}{$\begin{array}{c}\text { Probabilidad } \\
\text { Binomial }\end{array}$} & \multirow[t]{4}{*}{ conocimiento } & \multirow[t]{4}{*}{ conocimiento } & Bastante (2) & 24 & $39.34 \%$ \\
\hline & & & Regular (3) & 2 & $3.28 \%$ \\
\hline & & & Poco (4) & 0 & $0.00 \%$ \\
\hline & & & Nada (5) & 0 & $0.00 \%$ \\
\hline & \multirow{6}{*}{$\begin{array}{l}\text { Desarrollo } \\
\text { de } \\
\text { habilidades } \\
\text { matemáticas }\end{array}$} & \multirow{6}{*}{$\begin{array}{l}\text { 8. La aplicación } \\
\text { GeoGebra facilita el } \\
\text { desarrollo de las } \\
\text { habilidades } \\
\text { matemáticas }\end{array}$} & & & \\
\hline & & & Totalmente (1) & 39 & $63.93 \%$ \\
\hline & & & Bastante (2) & 22 & $36.07 \%$ \\
\hline & & & Regular (3) & 0 & $0.00 \%$ \\
\hline & & & Poco (4) & 0 & $0.00 \%$ \\
\hline & & & Nada (5) & 0 & $0.00 \%$ \\
\hline & \multirow{6}{*}{ Rol activo } & \multirow{6}{*}{$\begin{array}{l}\text { 9. La aplicación } \\
\text { GeoGebra facilita el } \\
\text { rol activo del alumno }\end{array}$} & & & \\
\hline & & & Totalmente (1) & 46 & $75.41 \%$ \\
\hline & & & Bastante (2) & 12 & $19.67 \%$ \\
\hline & & & Regular (3) & 2 & $3.28 \%$ \\
\hline & & & Poco (4) & 1 & $1.64 \%$ \\
\hline & & & Nada (5) & 0 & $0.00 \%$ \\
\hline
\end{tabular}

Tabla 3. Cuestionario

Fuente: Elaboración propia

Por otro lado, las variables utilizadas en el enfoque cualitativo (preguntas abiertas) son el proceso de enseñanza-aprendizaje (Probabilidad Binomial, Asimilación del conocimiento y Simulación de datos), el empleo de la aplicación GeoGebra (Motivación, Beneficios y Satisfacción) y la Usabilidad de la aplicación GeoGebra (Diseño, Herramienta innovadora y Utilidad).

El Factor de Carga, Alfa de Cronbach, Average Variance Extracted (AVE) y Composite Reliability (CR) son utilizados para validar el instrumento de medición (Ver Tabla 4). Para garantizar la fiabilidad del instrumento de medición, el valor sobre el Alfa de Cronbach debe ser superior al 0.70 (Jaffar y Musa, 2019). En particular, las variables de Aplicación GeoGebra (0.746) y Proceso educativo sobre la Probabilidad Binomial (0.725) tienen un valor superior al 0.724 para el Alfa de Cronbach. 


\begin{tabular}{|c|c|c|c|c|c|c|}
\hline No. & Variable & Dimensión & $\begin{array}{l}\text { Factor de } \\
\text { Carga }\end{array}$ & $\begin{array}{l}\text { Alfa de } \\
\text { Cronbach }\end{array}$ & $\begin{array}{c}\text { Average Variance } \\
\text { Extracted }\end{array}$ & $\begin{array}{l}\text { Composite } \\
\text { Reliability }\end{array}$ \\
\hline \multirow{3}{*}{1} & \multirow{3}{*}{$\begin{array}{l}\text { Aplicación } \\
\text { GeoGebra }\end{array}$} & Contenidos & 0.921 & \multirow{3}{*}{0.746} & \multirow{3}{*}{0.683} & \multirow{3}{*}{0.864} \\
\hline & & Estética & 0.831 & & & \\
\hline & & Simulación & 0.715 & & & \\
\hline \multirow{3}{*}{2} & \multirow{3}{*}{$\begin{array}{c}\text { Proceso } \\
\text { educativo } \\
\text { sobre la } \\
\text { Probabilidad } \\
\text { Binomial }\end{array}$} & $\begin{array}{l}\text { Asimilación } \\
\text { del } \\
\text { conocimiento }\end{array}$ & 0.824 & \multirow{3}{*}{0.725} & \multirow{3}{*}{0.653} & \multirow{3}{*}{0.849} \\
\hline & & $\begin{array}{l}\text { Desarrollo } \\
\text { de } \\
\text { habilidades } \\
\text { matemáticas }\end{array}$ & 0.829 & & & \\
\hline & & Rol activo & 0.770 & & & \\
\hline
\end{tabular}

Tabla 4. Validación del instrumento de medición

Fuente: Elaboración propia

Para validar el instrumento de medición, el valor del Composite Reliability debe superar al 0.70 (Jaffar y Musa, 2019). En este caso, las variables de la Aplicación GeoGebra (0.864) y el Proceso educativo sobre la Probabilidad Binomial (0.849) cumplen con este criterio (Ver Tabla 4).

Asimismo, los factores de carga deben presentar un valor superior al 0.5 (Jaffar y Musa, 2019). Los valores de carga para las dimensiones del cuestionario sobre el uso de la Aplicación GeoGebra son superiores al 0.70, esto es, Contenidos (0.921), Estética (0.831), Simulación (0.715), Asimilación del conocimiento $(0.824)$, Desarrollo de habilidades matemáticas $(0.829)$ y Rol activo $(0.770)$.

\section{d. Análisis de datos}

En el mes de abril del 2018, el instrumento de medición (cuestionario) es aplicado a los alumnos de la asignatura Instrumentación estadística para los negocios con la finalidad de analizar el impacto de la aplicación GeoGebra en el proceso de enseñanza-aprendizaje sobre la Probabilidad Binomial por medio de la hoja de cálculo (regresión lineal), el software Maxqda (análisis cualitativo) y la herramienta Rapidminer (modelos predictivos y aprendizaje automático).

El aprendizaje automático (machine learning) permite realizar el cálculo de regresiones lineales por medio de la segmentación de la muestra. La muestra de entrenamiento $(60 \%, 70 \%$ y $80 \%)$ permite establecer las regresiones lineales con el propósito de analizar el impacto de la aplicación GeoGebra en el proceso de enseñanza-aprendizaje y la muestra de evaluación (40\%, 30\% y 20\%) permite identificar la exactitud y eficiencia de estas regresiones lineales.

La Figura 2 muestra el uso de la herramienta Rapidminer para realizar el cálculo del aprendizaje automático con $60 \%, 70 \%$ y $80 \%$ de entrenamiento (regresión lineal). Cabe mencionar que el componente Split Data permite establecer los valores de entrenamiento y evaluación. 


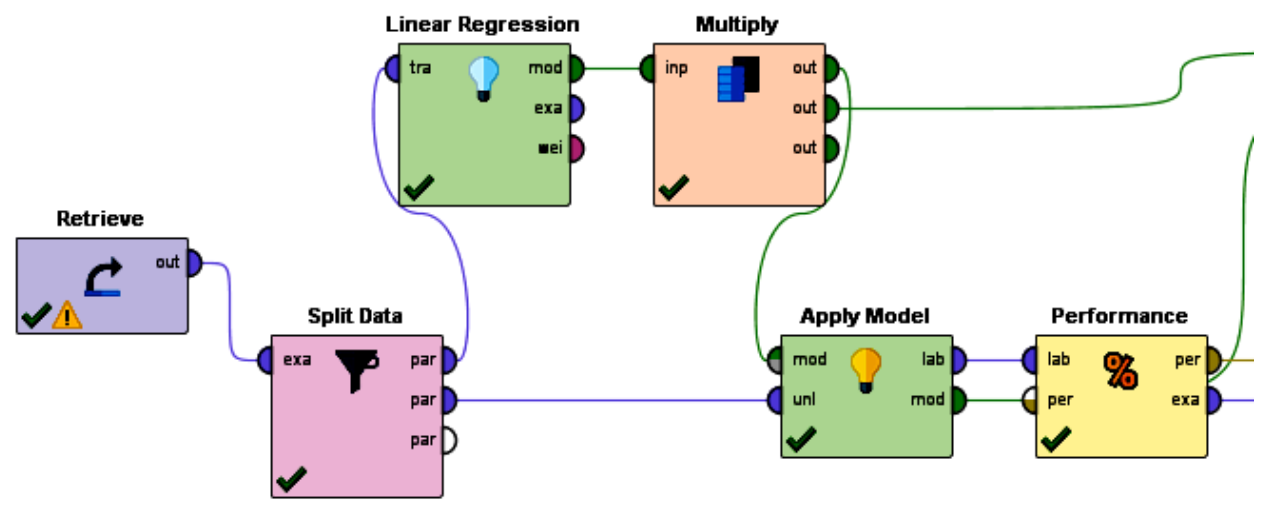

Figura 2. Aprendizaje automático en la herramienta Rapidminer

Fuente: Elaboración propia por medio de la herramienta Rapidminer

La ciencia de datos permite identificar las relaciones ocultas entre las variables con la finalidad de establecer diversos modelos y clasificaciones predictivas. En particular, esta investigación utiliza la técnica árbol de decisión para encontrar diversos modelos predictivos sobre la aplicación GeoGebra (contenidos, estética y simulación), el perfil del estudiante (género, edad y carrera) y el proceso de enseñanza-aprendizaje.

Por ejemplo, la Figura 3 muestra la información utilizada para la construcción del Modelo predictivo 1 sobre los contenidos de la aplicación GeoGebra y la asimilación del conocimiento sobre la Probabilidad Binomial por medio de la herramienta Rapidminer.

\begin{tabular}{|l|l|l|l|l|l|}
\hline Row No. & Asimilación - & Género & Edad & Carrera & Contenidos \\
\hline $\mathbf{1}$ & Totalmente & Hombre & 20 & Contaduría & Totalmente \\
\hline 2 & Totalmente & Mujer & 19 & Comercio & Totalmente \\
\hline 3 & Totalmente & Mujer & 18 & Contaduría & Bastante \\
\hline $\mathbf{4}$ & Bastante & Mujer & 19 & Comercio & Bastante \\
\hline 5 & Bastante & Mujer & 19 & Mercadotecnia & Totalmente \\
\hline
\end{tabular}

Figura 3. Información para el Modelo predictivo 1

Fuente: Elaboración propia por medio de la herramienta Rapidminer

La Figura 4 presenta el uso de la herramienta Rapidminer para construir los modelos predictivos por medio de la técnica árbol de decisión (minería de datos). 


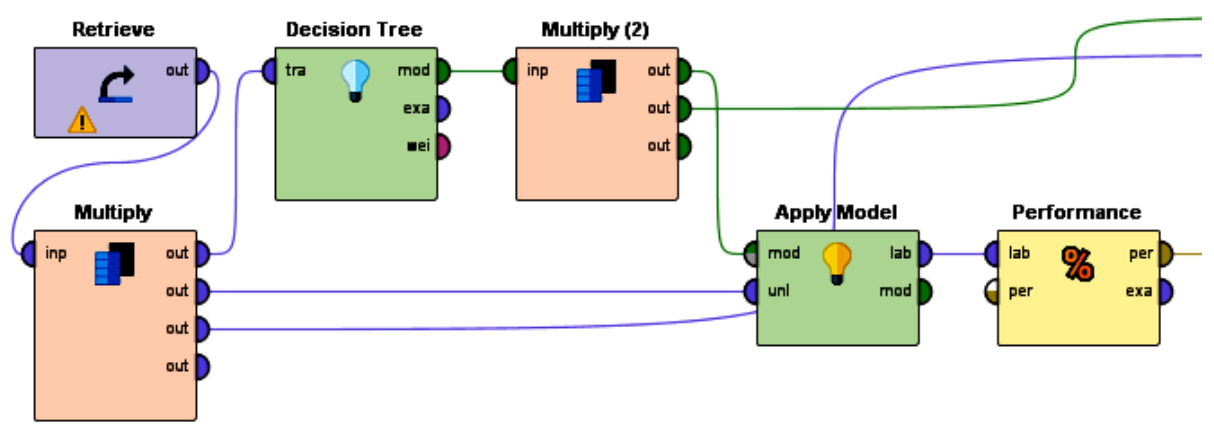

Figura 4. Modelos predictivos en la herramienta Rapidminer

Fuente: Elaboración propia por medio de la herramienta Rapidminer

Por otro lado, la herramienta Maxqda permite realizar el análisis cualitativo sobre el impacto de la aplicación GeoGebra en el proceso educativo sobre la Probabilidad Binomial (nube de palabras) por medio de las siguientes variables:

- Proceso de enseñanza-aprendizaje: Aprendizaje de la Probabilidad Binomial, Asimilación del conocimiento y Simulación de datos

- Empleo de la aplicación GeoGebra: Motivación, Beneficios de la aplicación y Satisfacción

- Usabilidad de la aplicación GeoGebra: Diseño de la aplicación, Herramienta Innovadora y Utilidad

\section{Resultados}

A continuación, se presentan los resultados sobre el impacto de la aplicación GeoGebra (contenidos, estética y simulación) en el proceso de enseñanza-aprendizaje sobre la Probabilidad Binomial considerando la ciencia de datos y el aprendizaje automático.

La mayoría de los estudiantes consideran que la aplicación GeoGebra facilita totalmente y bastante la asimilación de conocimiento, el desarrollo de las habilidades matemáticas y el rol activo del alumno (Ver Tabla 3). Por ejemplo, la aplicación GeoGebra facilita totalmente $(n=35,57.38 \%)$, bastante $(n=24,39.34 \%)$ y regular $(n=2,3.28 \%)$ la asimilación de conocimiento.

Asimismo, la aplicación GeoGebra facilita totalmente $(n=39,63.93 \%)$ y bastante $(n=22,36.07 \%)$ el desarrollo de las habilidades matemáticas. Incluso, la aplicación GeoGebra facilita totalmente $(n=46,75.41 \%)$, bastante $(n=12,19.67 \%)$, regular $(n=2,3.28 \%)$ y poco $(n=1,1.64 \%)$ el rol activo del alumno.

\section{a. Contenidos de la aplicación GeoGebra}

Los contenidos de GeoGebra facilitan totalmente $(n=40,65.57 \%)$ y bastante $(n=21,34.43 \%)$ el proceso de enseñanza-aprendizaje (Ver Tabla 3). Los resultados sobre la regresión lineal (hoja de cálculo) muestran que las Hipótesis 1, 2 y 3 sobre el impacto de aplicación GeoGebra (contenidos) en el proceso educativo son aceptadas (Ver Tabla 5). En particular, los contenidos de la aplicación GeoGebra influyen positivamente la asimilación del conocimiento (0.679), el desarrollo de las 
habilidades matemáticas $(0.466)$ y el rol activo $(0.541)$.

\begin{tabular}{|c|l|c|c|}
\hline No. & \multicolumn{1}{|c|}{ Hipótesis } & $\begin{array}{c}\text { Valor de la } \\
\text { regresión lineal }\end{array}$ & Conclusión \\
\hline 1 & $\begin{array}{l}\text { H1: Contenidos de GeoGebra Asimilación del } \\
\text { conocimiento }\end{array}$ & 0.679 & Aceptada \\
\hline 2 & $\begin{array}{l}\text { H2: Contenidos de GeoGebra Desarrollo de } \\
\text { habilidades matemáticas }\end{array}$ & 0.466 & Aceptada \\
\hline 3 & H3: Contenidos de GeoGebra Rol activo & 0.541 & Aceptada \\
\hline
\end{tabular}

Tabla 5. Resultados sobre los contenidos de la aplicación GeoGebra

Fuente: Elaboración propia

Los resultados del aprendizaje automático con $60 \%$, $70 \%$ y $80 \%$ de entrenamiento indican que los contenidos de la aplicación GeoGebra influyen positivamente el proceso educativo sobre la Probabilidad Binomial (Ver Tabla 6). Por ejemplo, el aprendizaje automático con 60\% de entrenamiento y $40 \%$ de evaluación indican que la hipótesis 1 (0.782), la hipótesis $2(0.338)$ y la hipótesis $3(0.440)$ son aceptadas. Del mismo modo, la hipótesis 1 , hipótesis 2 y hipótesis 3 son aceptadas a través del aprendizaje automático con $70 \%$ y $80 \%$ de entrenamiento.

\begin{tabular}{|l|c|c|c|c|}
\hline \multicolumn{1}{|c|}{ Hipótesis } & Entrenamiento & Evaluación & Regresión Lineal & Conclusión \\
\hline $\begin{array}{l}\text { H1: Contenidos de } \\
\text { GeoGebra }\end{array}$ & $60 \%$ & $40 \%$ & $y=0.782 x+0.434$ & Aceptada: 0.782 \\
\cline { 2 - 5 } $\begin{array}{l}\text { Asimilación del } \\
\text { conocimiento }\end{array}$ & $70 \%$ & $30 \%$ & $y=0.754 x+0.423$ & Aceptada: 0.754 \\
\cline { 2 - 5 } & $80 \%$ & $20 \%$ & $y=0.750 x+0.442$ & Aceptada: 0.750 \\
\hline $\begin{array}{l}\text { H2: Contenidos de } \\
\begin{array}{l}\text { GeoGebra } \\
\text { Desarrollo de } \\
\text { habilidades } \\
\text { matemáticas }\end{array}\end{array}$ & $60 \%$ & $40 \%$ & $y=0.338 x+0.965$ & Aceptada: 0.338 \\
\cline { 2 - 5 } & $80 \%$ & $30 \%$ & $y=0.349 x+0.900$ & Aceptada: 0.349 \\
\hline \multirow{2}{*}{$\begin{array}{l}\text { H3: Contenidos de } \\
\text { GeoGebra Rol } \\
\text { activo }\end{array}$} & $70 \%$ & $30 \%$ & $y=0.426 x+0.680$ & Aceptada: 0.426 \\
\cline { 2 - 5 } & $80 \%$ & $20 \%$ & $y=0.569 x+0.526$ & Aceptada: 0.569 \\
\hline
\end{tabular}

Tabla 6. Resultados del aprendizaje automático sobre los contenidos de GeoGebra

Fuente: Elaboración propia 
La Figura 5 muestra el Modelo predictivo 1 sobre los contenidos de la aplicación GeoGebra y la asimilación del conocimiento sobre la Probabilidad Binomial. Por ejemplo, si el estudiante considera que los contenidos de GeoGebra facilitan bastante el proceso de enseñanza-aprendizaje y tiene una edad mayor a 18.5 años entonces esta aplicación facilita bastante la asimilación de conocimiento.

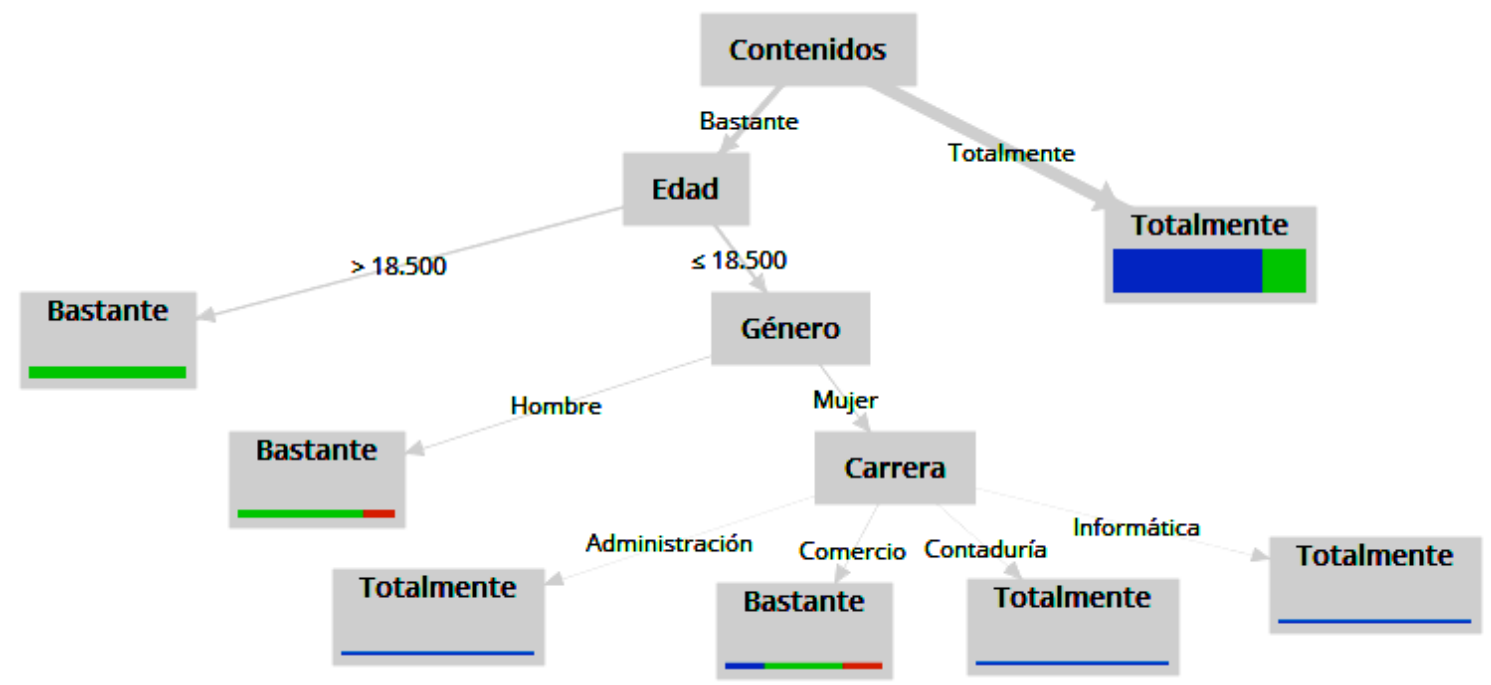

Figura 5. Modelo predictivo 1 sobre la aplicación GeoGebra en el proceso educativo

Fuente: Elaboración propia por medio de la herramienta Rapidminer

La Tabla 7 muestra las condiciones necesarias para que los contenidos de la aplicación GeoGebra faciliten totalmente la asimilación del conocimiento sobre la Probabilidad Binomial.

\begin{tabular}{|c|c|c|c|c|c|}
\hline \multirow{2}{*}{ No. } & \multicolumn{4}{|c|}{ Condiciones } & Resultados \\
\cline { 2 - 6 } & Contenidos & Edad & Carrera & Género & Asimilación del conocimiento \\
\hline 1 & Bastante & $\leq 18.5$ años & Administración & Mujer & Totalmente \\
\hline 2 & Bastante & $\leq 18.5$ años & Contaduría & Mujer & Totalmente \\
\hline 3 & Bastante & $\leq 18.5$ años & Informática & Mujer & Totalmente \\
\hline 4 & Totalmente & - & - & - & Totalmente \\
\hline
\end{tabular}

Tabla 7. Trayectorias del Modelo predictivo 1

Fuente: Elaboración propia 
La exactitud del Modelo predictivo 1 es 80.33\% (Ver Figura 6).

\begin{tabular}{|l|l|l|l|l|}
\hline \multicolumn{2}{|c|}{ accuracy: $\mathbf{8 0 . 3 3 \%}$} & true Totalmente & true Bastante & true Regular \\
\hline & 34 & 9 & 0 & $79.07 \%$ \\
\hline pred. Totalmente & 1 & 15 & 2 & $83.33 \%$ \\
\hline pred. Bastante & 0 & 0 & 0 & $0.00 \%$ \\
\hline pred. Regular & $97.14 \%$ & $62.50 \%$ & $0.00 \%$ & \\
\hline class recall & & 9 & \\
\hline
\end{tabular}

Figura 6. Exactitud del Modelo predictivo 1

Fuente: Elaboración propia por medio de la herramienta Rapidminer

La Figura 7 muestra el Modelo predictivo 2 sobre los contenidos de la aplicación GeoGebra y el desarrollo de las habilidades matemáticas. Por ejemplo, si el alumno piensa que los contenido de GeoGebra facilita bastante el proceso de enseñanza-aprendizaje, tiene una edad menor e igual que 19.5 años y estudia la carrera de Contaduría entonces esta aplicación facilita totalmente el desarrollo de las habilidades matemáticas.

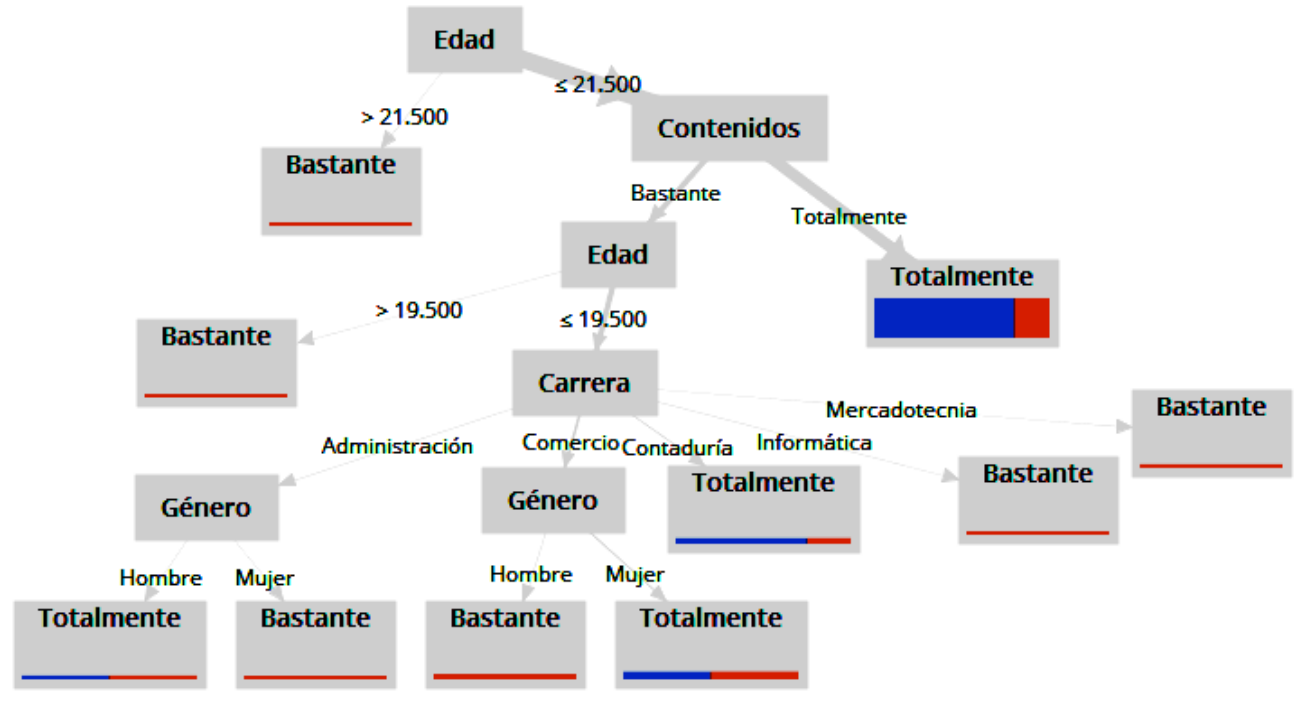

Figura 7. Modelo predictivo 2 sobre la aplicación GeoGebra en el proceso educativo

Fuente: Elaboración propia por medio de la herramienta Rapidminer

La Tabla 8 muestra las condiciones necesarias para que los contenidos de la aplicación GeoGebra faciliten totalmente el desarrollo de las habilidades sobre la Probabilidad Binomial. 


\begin{tabular}{|c|c|c|c|c|c|}
\hline \multirow{2}{*}{ No. } & \multicolumn{4}{|c|}{ Condiciones } & Resultados \\
\cline { 2 - 6 } & Contenidos & Edad & Carrera & Género & $\begin{array}{c}\text { Desarrollo de habilidades } \\
\text { matemáticas }\end{array}$ \\
\hline 1 & Totalmente & $\leq 21.5$ años & - & - & Totalmente \\
\hline 2 & Bastante & $\leq 19.5$ años & Administración & Hombre & Totalmente \\
\hline 3 & Bastante & $\leq 19.5$ años & Comercio & Mujer & Totalmente \\
\hline 4 & Bastante & $\leq 19.5$ años & Contaduría & - & Totalmente \\
\hline
\end{tabular}

Tabla 8. Trayectorias del Modelo predictivo 2

Fuente: Elaboración propia

La exactitud del Modelo predictivo 2 es 78.69\% (Ver Figura 8).

\begin{tabular}{|c|c|c|c|}
\hline & true Totalmente & true Bastante & class precision \\
\hline pred. Totalmente & 35 & 9 & $79.55 \%$ \\
\hline pred. Bastante & 4 & 13 & $76.47 \%$ \\
\hline class recall & $89.74 \%$ & $59.09 \%$ & \\
\hline
\end{tabular}

Figura 8. Exactitud del Modelo predictivo 2

Fuente: Elaboración propia por medio de la herramienta Rapidminer

La Figura 9 muestra el Modelo predictivo 3 sobre los contenidos de la aplicación GeoGebra y el rol activo del estudiante. Por ejemplo, si el estudiante considera que los contenidos de GeoGebra facilitan totalmente el proceso de enseñanza-aprendizaje y tiene una edad menor e igual a 21.5 años entonces esta aplicación facilita totalmente el rol activo del alumno.

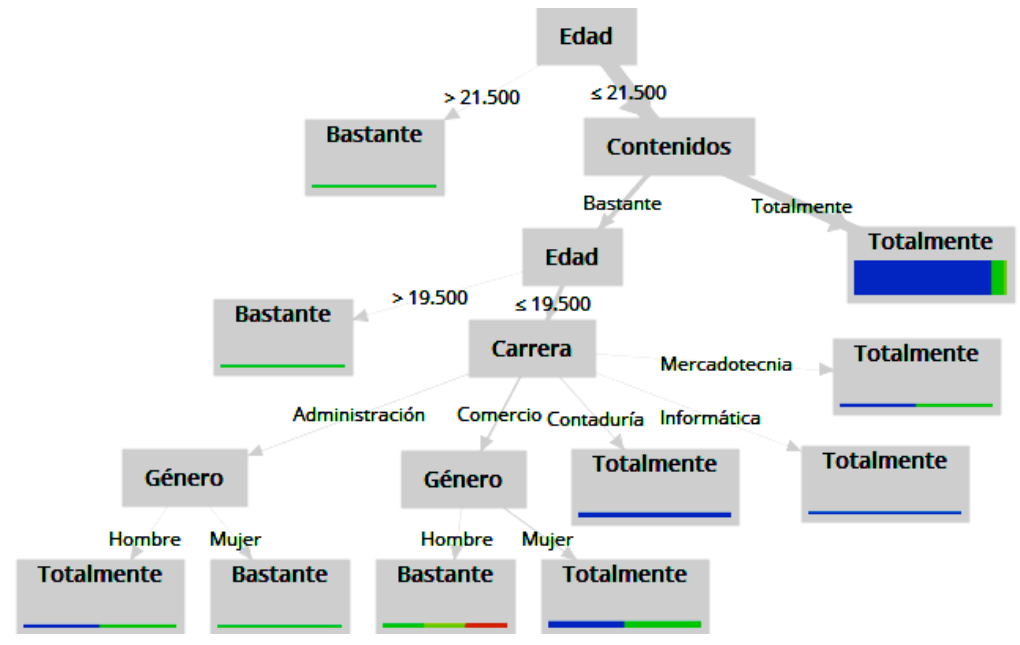

Figura 9. Modelo predictivo 3 sobre la aplicación GeoGebra en el proceso educativo Fuente: Elaboración propia por medio de la herramienta Rapidminer 
La Tabla 9 muestra las condiciones necesarias para que los contenidos de la aplicación GeoGebra facilite totalmente el rol activo del estudiante.

\begin{tabular}{|c|c|c|c|c|c|}
\hline \multirow{2}{*}{ No. } & \multicolumn{4}{|c|}{ Condiciones } & Resultados \\
\cline { 2 - 6 } & Contenidos & Edad & Carrera & Género & Rol activo \\
\hline 1 & Totalmente & $\leq 21.5$ años & & & Totalmente \\
\hline 2 & Bastante & $\leq 19.5$ años & Administración & Hombre & Totalmente \\
\hline 3 & Bastante & $\leq 19.5$ años & Comercio & Mujer & Totalmente \\
\hline 4 & Bastante & $\leq 19.5$ años & Contaduría & - & Totalmente \\
\hline 5 & Bastante & $\leq 19.5$ años & Informática & - & Totalmente \\
\hline 6 & Bastante & $\leq 19.5$ años & Mercadotecnia & - & Totalmente \\
\hline
\end{tabular}

Tabla 9. Trayectorias del Modelo predictivo 3

Fuente: Elaboración propia

La exactitud del Modelo predictivo 3 es $81.97 \%$ (Ver Figura 10).

\begin{tabular}{|l|l|l|l|l|l|}
\hline \multicolumn{1}{|c|}{ accuracy: 81.97\% } & true Totalmente & true Bastante & true Regular & true Poco & class precision \\
\hline pred. Totalmente & 42 & 4 & 1 & 0 & $89.36 \%$ \\
\hline pred. Bastante & 4 & 8 & 1 & 1 & $57.14 \%$ \\
\hline pred. Regular & 0 & 0 & 0 & 0 & $0.00 \%$ \\
\hline pred. Poco & 0 & 0 & 0 & 0 & $0.00 \%$ \\
\hline class recall & $91.30 \%$ & $66.67 \%$ & $0.00 \%$ & $0.00 \%$ & \\
\hline
\end{tabular}

Figura 10. Exactitud del Modelo predictivo 3

Fuente: Elaboración propia por medio de la herramienta Rapidminer

\section{b. Estética de la aplicación GeoGebra}

La estética de GeoGebra facilita totalmente $(n=40,65.57 \%)$, bastante $(n=16,26.23 \%)$ y regular $(n=5,8.20 \%)$ el proceso de enseñanza-aprendizaje (Ver Tabla 3). Los resultados sobre la regresión lineal (hoja de cálculo) muestran que las Hipótesis 4, 5 y 6 sobre el impacto de la aplicación GeoGebra en el proceso educativo son aceptadas (Ver Tabla 10). Por consiguiente, la estética de la aplicación GeoGebra influye positivamente la asimilación del conocimiento (0.403), el desarrollo de las habilidades matemáticas (0.305) y el rol activo (0.317). 


\begin{tabular}{|c|l|c|c|}
\hline No. & \multicolumn{1}{|c|}{ Hipótesis } & \multicolumn{1}{|c|}{$\begin{array}{c}\text { Valor de la } \\
\text { regresión lineal }\end{array}$} & Conclusión \\
\hline 1 & $\begin{array}{l}\text { H4: Estética de GeoGebra Asimilación del } \\
\text { conocimiento }\end{array}$ & 0.403 & Aceptada \\
\hline 2 & $\begin{array}{l}\text { H5: Estética de GeoGebra Desarrollo de habilidades } \\
\text { matemáticas }\end{array}$ & 0.305 & Aceptada \\
\hline 3 & H6: Estética de GeoGebra Rol activo & 0.317 & Aceptada \\
\hline
\end{tabular}

Tabla 10. Resultados sobre la estética de la aplicación GeoGebra

Fuente: Elaboración propia

Los resultados del aprendizaje automático con $60 \%, 70 \%$ y $80 \%$ de entrenamiento indican que la estética de la aplicación GeoGebra influyen positivamente el proceso educativo sobre la Probabilidad Binomial (Ver Tabla 11). Por ejemplo, el aprendizaje automático con $60 \%$ de entrenamiento y $40 \%$ de evaluación indican que la hipótesis $4(0.391)$, la hipótesis $5(0.186)$ y la hipótesis $6(0.385)$ son aceptadas. Del mismo modo, la hipótesis 4, hipótesis 5 y hipótesis 6 son aceptadas a través del aprendizaje automático con $70 \%$ y $80 \%$ de entrenamiento.

\begin{tabular}{|c|c|c|c|c|}
\hline Hipótesis & Entrenamiento & Evaluación & Regresión Lineal & Conclusión \\
\hline \multirow{3}{*}{$\begin{array}{l}\text { H4: Estética de } \\
\text { GeoGebra Asimilación } \\
\text { del conocimiento }\end{array}$} & $60 \%$ & $40 \%$ & $y=0.391 x+0.931$ & Aceptada: 0.391 \\
\hline & $70 \%$ & $30 \%$ & $y=0.408 x+0.852$ & Aceptada: 0.408 \\
\hline & $80 \%$ & $20 \%$ & $y=0.431 x+0.844$ & Aceptada: 0.431 \\
\hline \multirow{3}{*}{$\begin{array}{l}\text { H5: Estética de } \\
\text { GeoGebra } \quad \text { Desarrollo } \\
\text { de } \\
\text { matemáticas }\end{array}$} & $60 \%$ & $40 \%$ & $y=0.186 x+1.155$ & Aceptada: 0.186 \\
\hline & $70 \%$ & $30 \%$ & $y=0.211 x+1.067$ & Aceptada: 0.211 \\
\hline & $80 \%$ & $20 \%$ & $y=0.249 x+1.046$ & Aceptada: 0.249 \\
\hline \multirow{3}{*}{$\begin{array}{l}\text { H6: Estetica de } \\
\text { GeoGebra Rol activo }\end{array}$} & $60 \%$ & $40 \%$ & $y=0.385 x+0.724$ & Aceptada: 0.385 \\
\hline & $70 \%$ & $30 \%$ & $y=0.383 x+0.702$ & Aceptada: 0.383 \\
\hline & $80 \%$ & $20 \%$ & $Y=0.311 x+0.854$ & Aceptada: 0.311 \\
\hline
\end{tabular}

Tabla 11. Resultados del aprendizaje automático sobre la estética de GeoGebra

Fuente: Elaboración propia

La Figura 11 presenta el Modelo predictivo 4 sobre la estética de la aplicación GeoGebra y la asimilación del conocimiento sobre la Probabilidad Binomial. Por ejemplo, si el alumno piensa que la estética de GeoGebra facilita totalmente el proceso de enseñanza-aprendizaje, tiene una edad menor e igual a 19.5 años y estudia la carrera de Mercadotecnia entonces esta aplicación facilita totalmente la asimilación del conocimiento. 


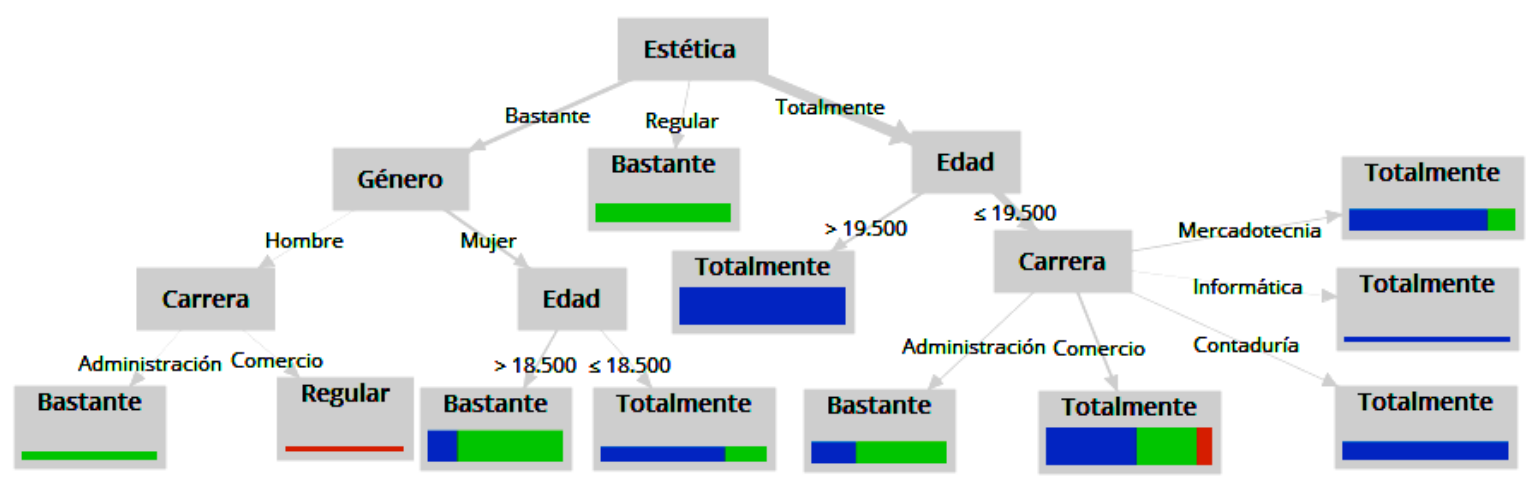

Figura 11. Modelo predictivo 4 sobre la aplicación GeoGebra en el proceso educativo

Fuente: Elaboración propia por medio de la herramienta Rapidminer

La Tabla 12 muestra las condiciones necesarias para que la estética de la aplicación GeoGebra facilite totalmente la asimilación del conocimiento sobre la Probabilidad Binomial.

\begin{tabular}{|c|c|c|c|c|c|}
\hline \multirow{2}{*}{ No. } & \multicolumn{4}{|c|}{ Condiciones } & Resultados \\
\cline { 2 - 6 } & Estética & Edad & Carrera & Género & Asimilación del conocimiento \\
\hline 1 & Bastante & $\leq 18.5$ años & - & Mujer & Totalmente \\
\hline 2 & Totalmente & $\geq 19.5$ años & - & - & Totalmente \\
\hline 3 & Totalmente & $\leq 19.5$ años & Comercio & - & Totalmente \\
\hline 4 & Totalmente & $\leq 19.5$ años & Contaduría & - & Totalmente \\
\hline 5 & Totalmente & $\leq 19.5$ años & Informática & - & Totalmente \\
\hline 6 & Totalmente & $\leq 19.5$ años & Mercadotecnia & - & Totalmente \\
\hline
\end{tabular}

Tabla 12. Trayectorias del Modelo predictivo 4

Fuente: Elaboración propia

La exactitud del Modelo predictivo 4 es $81.97 \%$ (Ver Figura 12).

\begin{tabular}{|l|l|l|l|l|}
\hline \multicolumn{2}{|l}{ accuracy: 81.97\% } & true Totalmente & true Bastante & true Regular \\
\hline & 31 & 6 & 1 & class precision \\
\hline pred. Totalmente & 4 & 18 & 0 & $81.58 \%$ \\
\hline pred. Bastante & 0 & 0 & 1 & $100.00 \%$ \\
\hline pred. Regular & $88.57 \%$ & $75.00 \%$ & $50.00 \%$ & \\
\hline class recall & & $61.82 \%$ & \\
\hline
\end{tabular}

Figura 12. Exactitud del Modelo predictivo 4

Fuente: Elaboración propia por medio de la herramienta Rapidminer 
La Figura 13 presenta el Modelo predictivo 5 sobre la estética de aplicación GeoGebra y el desarrollo de las habilidades matemáticas. Por ejemplo, si el alumno considera que la estética de GeoGebra facilita bastante el proceso de enseñanza-aprendizaje y tiene una edad menor e igual a 18.5 años entonces esta aplicación facilita totalmente el desarrollo de habilidades matemáticas.

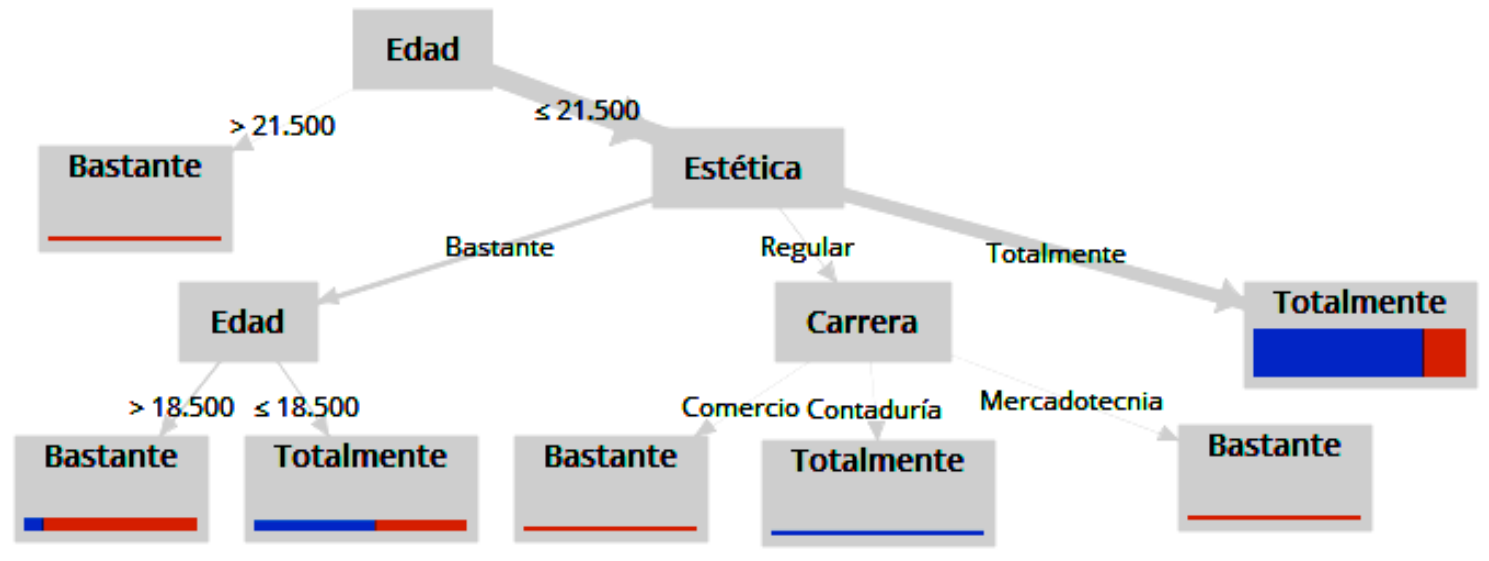

Figura 13. Modelo predictivo 5 sobre la aplicación GeoGebra en el proceso educativo Fuente: Elaboración propia por medio de la herramienta Rapidminer

La Tabla 13 muestra las condiciones necesarias para que la estética de la aplicación GeoGebra facilite totalmente el desarrollo de las habilidades matemáticas sobre la Probabilidad Binomial.

\begin{tabular}{|c|c|c|c|c|c|}
\hline \multirow{2}{*}{ No. } & \multicolumn{4}{|c|}{ Condiciones } & Resultados \\
\cline { 2 - 6 } & Estética & Edad & Carrera & Género & $\begin{array}{c}\text { Desarrollo de habilidades } \\
\text { matemáticas }\end{array}$ \\
\hline 1 & Bastante & $\leq 18.5$ años & - & - & Totalmente \\
\hline 2 & Regular & $\leq 21.5$ años & Contaduría & - & Totalmente \\
\hline 3 & Totalmente & $\leq 21.5$ años & - & - & Totalmente \\
\hline
\end{tabular}

Tabla 13. Trayectorias del Modelo predictivo 5

Fuente: Elaboración propia 
La exactitud del Modelo predictivo 5 es 80.33\% (Ver Figura 14).

\begin{tabular}{|l|l|l|l|}
\hline \multicolumn{1}{|c|}{ accuracy: $\mathbf{8 0 . 3 3 \%}$} & true Totalmente & true Bastante & class precision \\
\hline & 38 & 11 & $77.55 \%$ \\
\hline pred. Totalmente & 1 & 11 & $91.67 \%$ \\
\hline pred. Bastante & $97.44 \%$ & $50.00 \%$ & \\
\hline class recall & & & \\
\hline
\end{tabular}

Figura 14. Exactitud del Modelo predictivo 5

Fuente: Elaboración propia por medio de la herramienta Rapidminer

La Figura 15 presenta el Modelo Predictivo 6 sobre la estética de la aplicación GeoGebra y el rol activo del estudiante. Por ejemplo, si el alumno piensa que la estética de GeoGebra facilita totalmente el proceso de enseñanza-aprendizaje y tiene una edad menor e igual a 21.5 años entonces esta aplicación facilita totalmente el rol activo del estudiante.

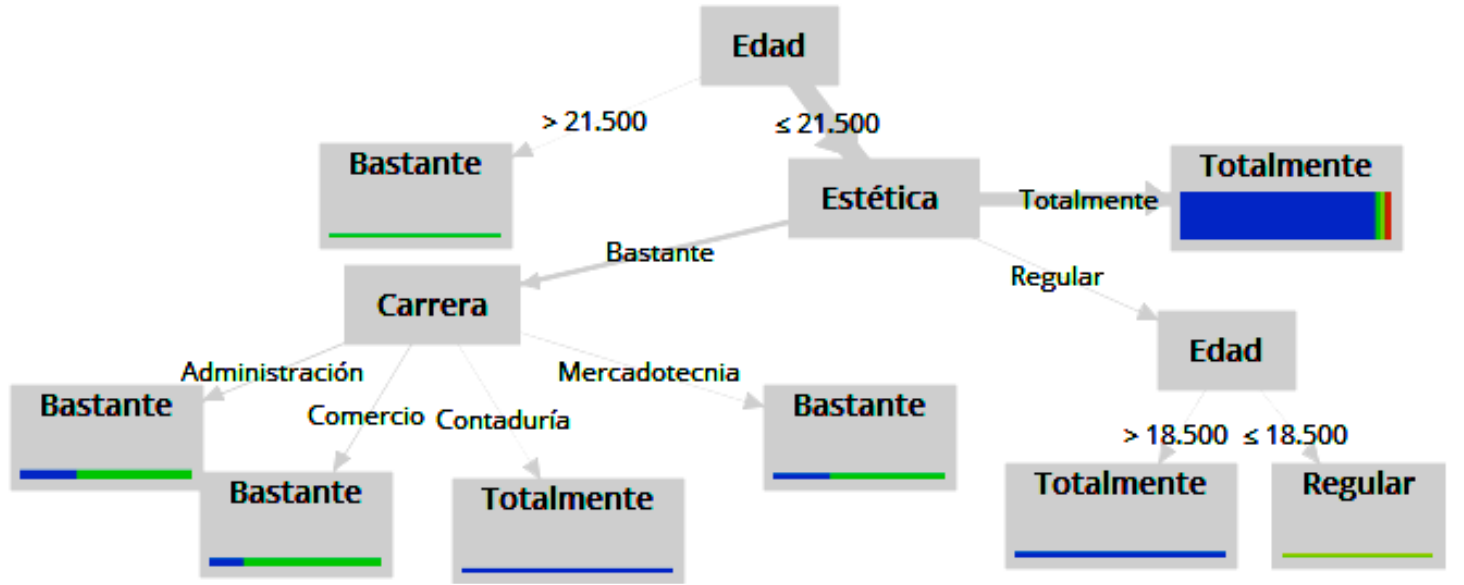

Figura 15. Modelo predictivo 6 sobre la aplicación GeoGebra en el proceso educativo

Fuente: Elaboración propia por medio de la herramienta Rapidminer

La Tabla 14 muestra las condiciones necesarias para que la estética de la aplicación GeoGebra facilite totalmente el rol activo del estudiante.

\begin{tabular}{|c|c|c|c|c|c|}
\hline \multirow{2}{*}{ No. } & \multicolumn{4}{|c|}{ Condiciones } & Resultados \\
\cline { 2 - 6 } & Estética & Edad & Carrera & Género & Rol activo \\
\hline 1 & Bastante & $\leq 21.5$ años & Contaduría & - & Totalmente \\
\hline 2 & Totalmente & $\leq 21.5$ años & - & - & Totalmente \\
\hline 3 & Regular & $\begin{array}{c}\leq 21.5 \text { años y } \\
>18.5 \text { años }\end{array}$ & - & - & Totalmente \\
\hline
\end{tabular}

Tabla 14. Trayectorias del Modelo predictivo 6

Fuente: Elaboración propia 
La exactitud del Modelo predictivo 6 es 88.52\% (Ver Figura 16).

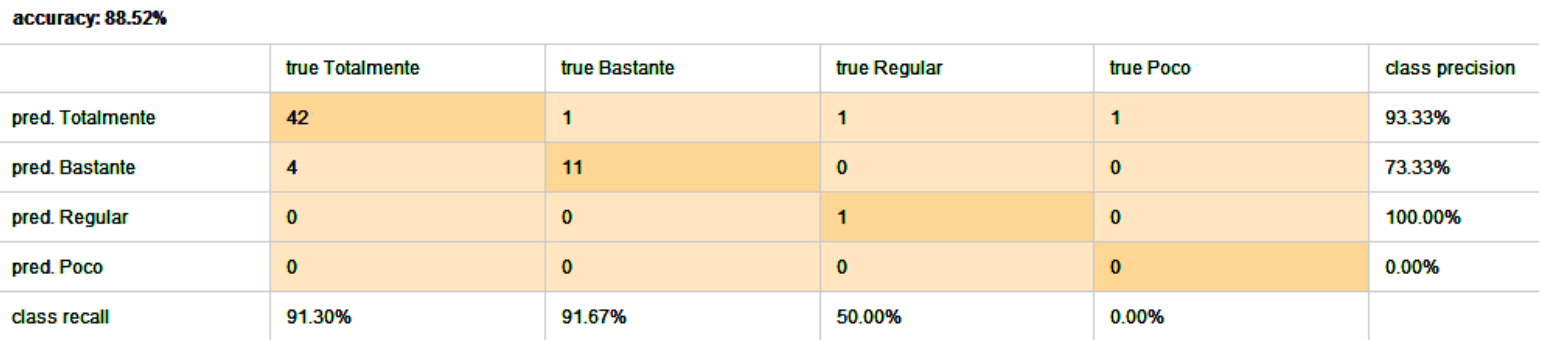

Figura 16. Exactitud del Modelo predictivo 6

Fuente: Elaboración propia por medio de la herramienta Rapidminer

\section{c. Simulación de la aplicación GeoGebra}

La simulación de GeoGebra facilita totalmente $(n=41,67.21 \%)$, bastante $(n=18,29.51 \%)$ y regular $(n=2,3.28 \%)$ el proceso de enseñanza-aprendizaje (Ver Tabla 3). Los resultados sobre la regresión lineal (hoja de cálculo) muestran que las Hipótesis 7, 8 y 9 sobre el impacto de la aplicación GeoGebra (simulación) son aceptadas (Ver Tabla 15). Por consiguiente, la simulación de la aplicación GeoGebra influye positivamente la asimilación del conocimiento (0.548), el desarrollo de las habilidades matemáticas $(0.501)$ y el rol activo $(0.450)$.

\begin{tabular}{|c|l|c|c|}
\hline No. & \multicolumn{1}{|c|}{ Hipótesis } & $\begin{array}{c}\text { Valor de la } \\
\text { regresión lineal }\end{array}$ & Conclusión \\
\hline 1 & $\begin{array}{l}\text { H7: Simulación de GeoGebra } \\
\text { conocimiento }\end{array}$ & 0.548 & Aceptada \\
\hline 2 & $\begin{array}{l}\text { H8: Simulación de GeoGebra desarrollo de del } \\
\text { habilidades matemáticas }\end{array}$ & 0.501 & Aceptada \\
\hline 3 & H9: Simulación de GeoGebra Rol activo & 0.450 & Aceptada \\
\hline
\end{tabular}

Tabla 15. Resultados sobre la simulación de la aplicación GeoGebra

Fuente: Elaboración propia

Los resultados del aprendizaje automático con $60 \%, 70 \%$ y $80 \%$ de entrenamiento indican que la simulación de la aplicación GeoGebra influyen positivamente el proceso educativo sobre la Probabilidad Binomial (Ver Tabla 16). Por ejemplo, el aprendizaje automático con $60 \%$ de entrenamiento y $40 \%$ de evaluación indican que la hipótesis $7(0.576)$, la hipótesis $8(0.413)$ y la hipótesis 9 (0.232) son aceptadas. Del mismo modo, la hipótesis 7 , hipótesis 8 y hipótesis 9 son aceptadas a través del aprendizaje automático con $70 \%$ y $80 \%$ de entrenamiento. 


\begin{tabular}{|c|c|c|c|c|}
\hline Hipótesis & Entrenamiento & Evaluación & Regresión Lineal & Conclusión \\
\hline \multirow{3}{*}{$\begin{array}{ll}\text { H7: Simulación } & \text { de } \\
\text { GeoGebra } & \\
\text { Asimilación } & \text { del } \\
\text { conocimiento } & \end{array}$} & $60 \%$ & $40 \%$ & $y=0.576 x+0.702$ & Aceptada: 0.576 \\
\hline & $70 \%$ & $30 \%$ & $y=0.626 x+0.596$ & Aceptada: 0.626 \\
\hline & $80 \%$ & $20 \%$ & $y=0.606 x+0.614$ & Aceptada: 0.606 \\
\hline \multirow{3}{*}{$\begin{array}{l}\text { H8: Simulación de } \\
\text { GeoGebra Desarrollo } \\
\text { de habilidades } \\
\text { matemáticas }\end{array}$} & $60 \%$ & $40 \%$ & $y=0.413 x+0.851$ & Aceptada: 0.413 \\
\hline & $70 \%$ & $30 \%$ & $y=0.460 x+0.596$ & Aceptada: 0.460 \\
\hline & $80 \%$ & $20 \%$ & $y=0.494 x+0.711$ & Aceptada: 0.494 \\
\hline \multirow{3}{*}{$\begin{array}{l}\text { H9: Simulación de } \\
\text { GeoGebra Rol activo }\end{array}$} & $60 \%$ & $40 \%$ & $y=0.232 x+0.970$ & Aceptada: 0.232 \\
\hline & $70 \%$ & $30 \%$ & $y=0.268 x+0.893$ & Aceptada: 0.268 \\
\hline & $80 \%$ & $20 \%$ & $y=0.497 x+0.605$ & Aceptada: 0.497 \\
\hline
\end{tabular}

Tabla 16. Resultados del aprendizaje automático sobre la simulación de GeoGebra

Fuente: Elaboración propia

La Figura 17 muestra el Modelo predictivo 7 sobre la simulación de la aplicación GeoGebra y la asimilación del conocimiento sobre la Probabilidad Binomial. Por ejemplo, si el estudiante piensa que la simulación de GeoGebra facilita totalmente el proceso de enseñanza-aprendizaje y tiene una edad menor e igual a 21.5 años entonces esta aplicación facilita totalmente la asimilación del conocimiento.

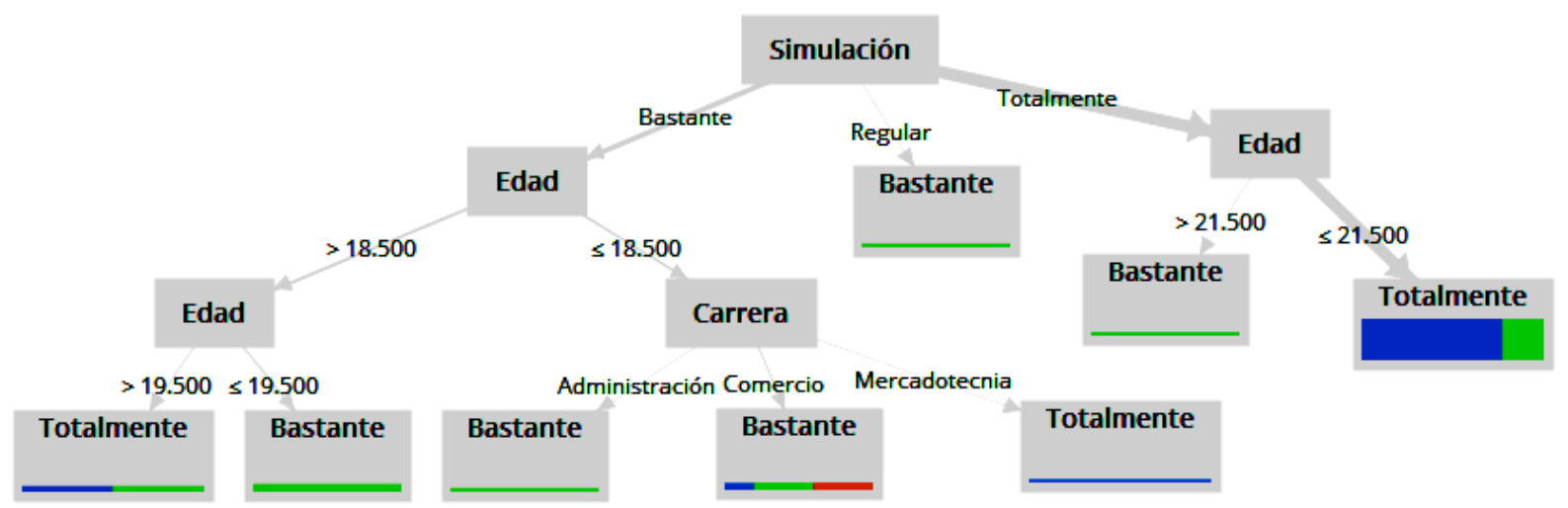

Figura 17. Modelo predictivo 7 sobre la aplicación GeoGebra en el proceso educativo

Fuente: Elaboración propia por medio de la herramienta Rapidminer

La Tabla 17 muestra las condiciones necesarias para que la simulación de la aplicación GeoGebra facilite totalmente la asimilación del conocimiento. 


\begin{tabular}{|c|c|c|c|c|c|}
\hline \multirow{2}{*}{ No. } & \multicolumn{4}{|c|}{ Condiciones } & Resultados \\
\cline { 2 - 6 } & Simulación & Edad & Carrera & Género & Asimilación del conocimiento \\
\hline 1 & Bastante & $>19.5$ años & - & - & Totalmente \\
\hline 2 & Bastante & $\leq 18.5$ años & Mercadotecnia & - & Totalmente \\
\hline 3 & Totalmente & $\leq 21.5$ años & - & - & Totalmente \\
\hline
\end{tabular}

Tabla 17. Trayectorias del Modelo predictivo 7

Fuente: Elaboración propia

La exactitud del Modelo predictivo 7 es 77.05\% (Ver Figura 18).

\begin{tabular}{|c|c|c|c|c|}
\hline & true Totalmente & true Bastante & true Regular & class precision \\
\hline pred. Totalmente & 34 & 11 & 0 & $75.56 \%$ \\
\hline pred. Bastante & 1 & 13 & 2 & $81.25 \%$ \\
\hline pred. Regular & 0 & 0 & 0 & $0.00 \%$ \\
\hline
\end{tabular}

Figura 18. Exactitud del Modelo predictivo 7

Fuente: Elaboración propia por medio de la herramienta Rapidminer

La Figura 19 muestra el Modelo predictivo 8 sobre la simulación de la aplicación GeoGebra y el desarrollo de las habilidades matemáticas. Por ejemplo, si el alumno piensa que la simulación de GeoGebra facilita totalmente el proceso de enseñanza-aprendizaje y tiene una edad menor e igual a 21.5 años entonces esta aplicación facilita totalmente el desarrollo de las habilidades.

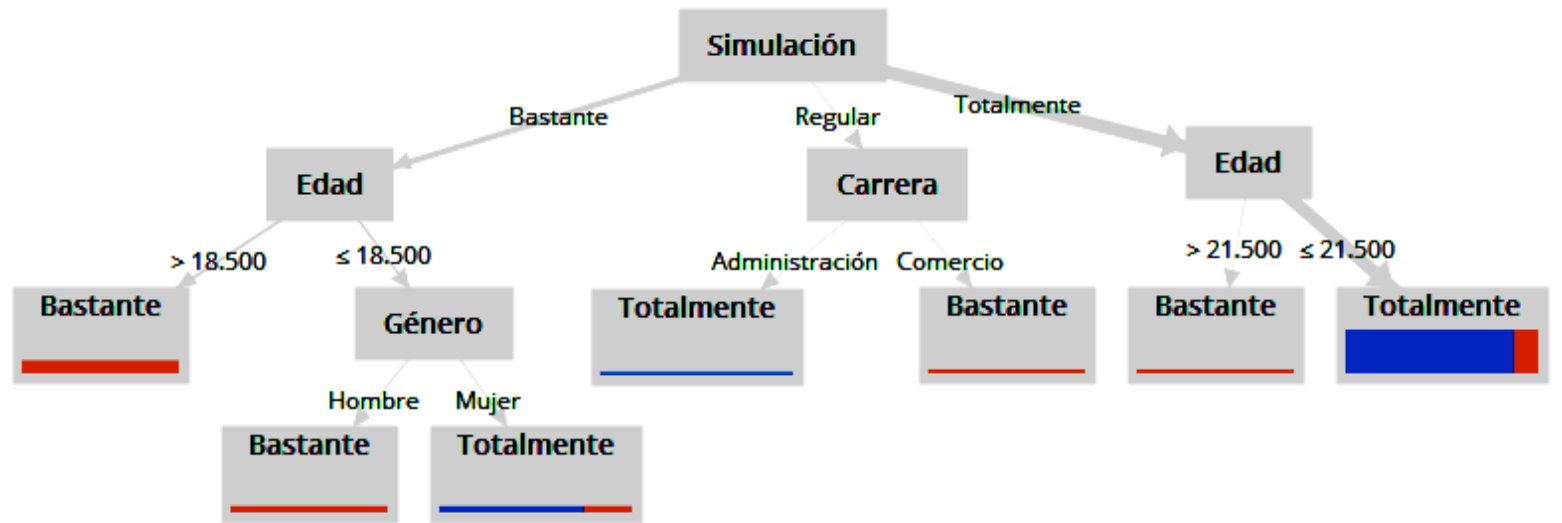

Figura 19. Modelo predictivo 8 sobre la aplicación GeoGebra en el proceso educativo

Fuente: Elaboración propia por medio de la herramienta Rapidminer

La Tabla 18 muestra las condiciones necesarias para que la simulación de la aplicación GeoGebra facilite totalmente el desarrollo de las habilidades matemáticas. 


\begin{tabular}{|c|c|c|c|c|c|}
\hline \multirow{2}{*}{ No. } & \multicolumn{3}{|c|}{ Condiciones } & Resultados \\
\cline { 2 - 6 } & Simulación & Edad & Carrera & Género & $\begin{array}{c}\text { Desarrollo de habilidades } \\
\text { matemáticas }\end{array}$ \\
\hline 1 & Bastante & $\leq 18.5$ años & - & Mujer & Totalmente \\
\hline 2 & Regular & - & Administración & - & Totalmente \\
\hline 3 & Totalmente & $\leq 21.5$ años & - & - & Totalmente \\
\hline
\end{tabular}

Tabla 18. Trayectorias del Modelo predictivo 8

Fuente: Elaboración propia

La exactitud del Modelo predictivo 8 es 90.16\% (Ver Figura 20).

\begin{tabular}{|c|c|c|c|}
\hline & true Totalmente & true Bastante & class precision \\
\hline pred. Totalmente & 39 & 6 & $86.67 \%$ \\
\hline pred. Bastante & 0 & 16 & $100.00 \%$ \\
\hline class recall & $100.00 \%$ & $72.73 \%$ & \\
\hline
\end{tabular}

Figura 20. Exactitud del Modelo predictivo 8

Fuente: Elaboración propia

La Figura 21 muestra el Modelo predictivo 9 sobre la simulación de la aplicación GeoGebra y el rol activo del estudiante. Por ejemplo, si el alumno considera que la simulación de GeoGebra facilita totalmente el proceso de enseñanza-aprendizaje y tiene una edad menor e igual a 21.5 años entonces esta aplicación facilita totalmente el rol activo del estudiante.

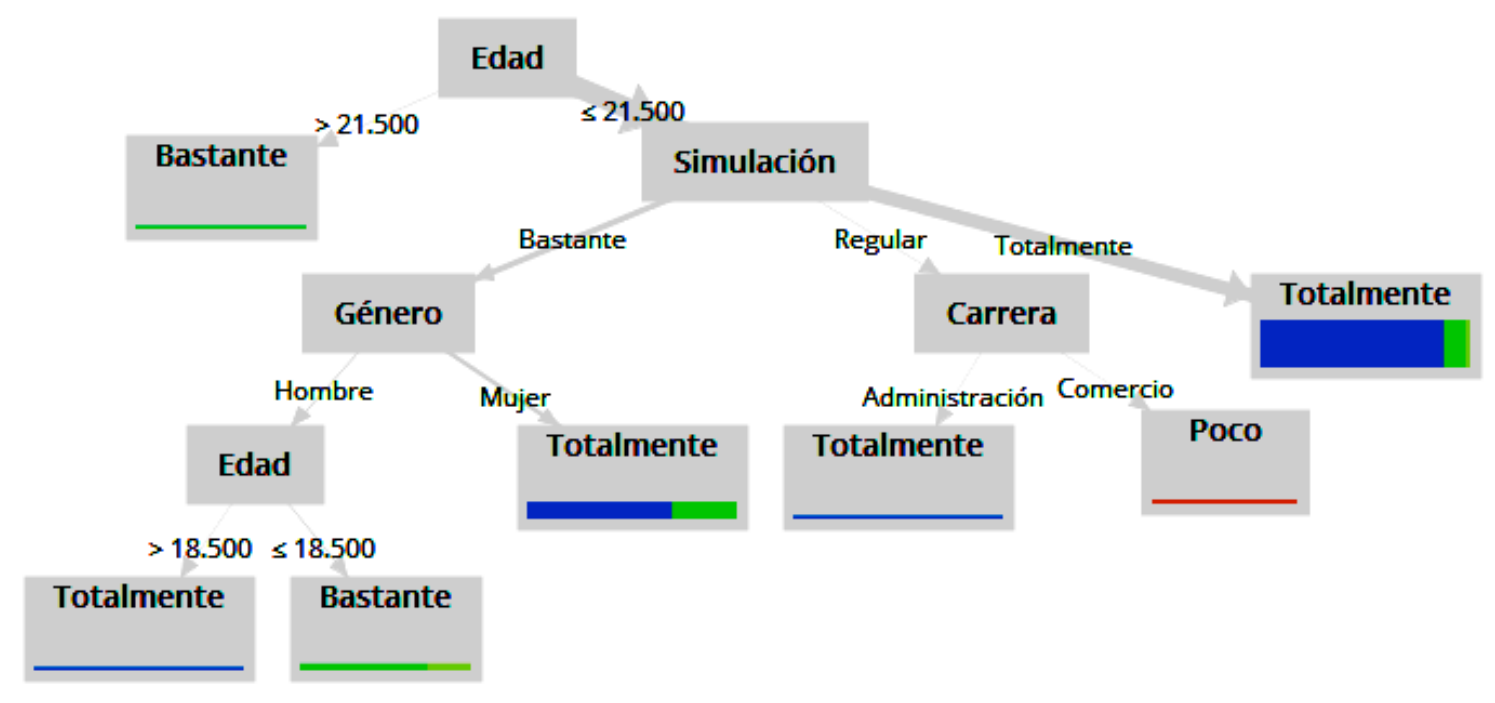

Figura 21. Modelo predictivo 9 sobre la aplicación GeoGebra en el proceso educativo

Fuente: Elaboración propia 
La Tabla 19 muestra las condiciones necesarias para que la simulación de la aplicación GeoGebra facilite totalmente el rol activo del estudiante en el aprendizaje.

\begin{tabular}{|c|c|c|c|c|c|}
\hline \multirow{2}{*}{ No. } & \multicolumn{4}{|c|}{ Condiciones } & Resultados \\
\cline { 2 - 6 } & Simulación & Edad & Carrera & Género & Rol activo \\
\hline 1 & Bastante & $\leq 21.5$ años & - & Mujer & Totalmente \\
\hline 2 & Totalmente & $\leq 21.5$ años & - & - & Totalmente \\
\hline 3 & Regular & $\leq 21.5$ años & Administración & - & Totalmente \\
\hline
\end{tabular}

Tabla 19. Trayectorias del Modelo predictivo 9

Fuente: Elaboración propia

La exactitud del Modelo predictivo 9 es del 83.61\% (Ver Figura 22).

\begin{tabular}{|l|l|l|l|l|l|}
\hline \multicolumn{1}{|c|}{ accuracy: 83.61\% } & true Totalmente & true Bastante & true Regular & true Poco & class precision \\
\hline pred. Totalmente & 46 & 8 & 1 & 0 & $83.64 \%$ \\
\hline pred. Bastante & 0 & 4 & 1 & 0 & $80.00 \%$ \\
\hline pred. Regular & 0 & 0 & 0 & 0 & $0.00 \%$ \\
\hline pred. Poco & 0 & 0 & 0 & 1 & $100.00 \%$ \\
\hline class recall & $100.00 \%$ & $33.33 \%$ & $0.00 \%$ & $0.00 \%$ \\
\hline
\end{tabular}

Figura 22. Exactitud del Modelo predictivo 9

Fuente: Elaboración propia

\section{d. Percepción de los estudiantes}

Para el análisis cualitativo, este estudio utilizó la herramienta Maxqda para construir la nube de palabras. Cabe mencionar que las variables analizadas son el Proceso de enseñanza-aprendizaje (Probabilidad Binomial, Asimilación del conocimiento y Simulación de datos), el Empleo de la aplicación GeoGebra (Motivación, Beneficios y Satisfacción) y la Usabilidad de la aplicación GeoGebra (Diseño, Herramienta Innovadora y Utilidad).

Los alumnos de la asignatura Instrumentación estadística para los negocios consideran que la aplicación GeoGebra facilita el proceso de enseñanza-aprendizaje sobre la Probabilidad Binomial:

- Sí, ayuda a la comprensión de los temas (Estudiante 28, Femenino, 18 años, Comercio).

- Sí, es más fácil el proceso (Estudiante 30, Masculino, 19 años, Administración).

- Sí, porque ayuda a comprender (Estudiante 41, Masculino, 19 años, Comercio).

De hecho, los estudiantes de las Licenciaturas en Administración, Contaduría, Comercio, Informática y Mercadotecnia señalan que la aplicación GeoGebra mejora la asimilación del conocimiento debido a que esta herramienta tecnológica permite aclarar las dudas y repasar los temas:

Sí, porque ayuda a aclarar las dudas (Estudiante 28, Femenino, 18 años, Comercio). 
- Sí, debido a que se puede practicar (Estudiante 33, Femenino, 20 años, Mercadotecnia).

- Sí, porque repasas tus conocimientos (Estudiante 50, Femenino, 18 años, Contaduría).

Por otro lado, la simulación de los datos en la aplicación GeoGebra ayuda a la comprensión de los temas sobre la Probabilidad Binomial por medio de las gráficas:

- Sí, porque ves los resultados exactos y con gráficas (Estudiante 25, Femenino, 20 años, Administración).

- Sí, de esta manera es más fácil comprender la información (Estudiante 28, Femenino, 18 años, Comercio).

- Sí, ya que las gráficas y tablas son claras (Estudiante 33, Femenino, 20 años, Mercadotecnia).

Las palabras más frecuentes para la variable Proceso de enseñanza-aprendizaje son Fácil, Ayuda, Entender, Proceso, Aprender, Comprender y Dudas (Ver Figura 23).

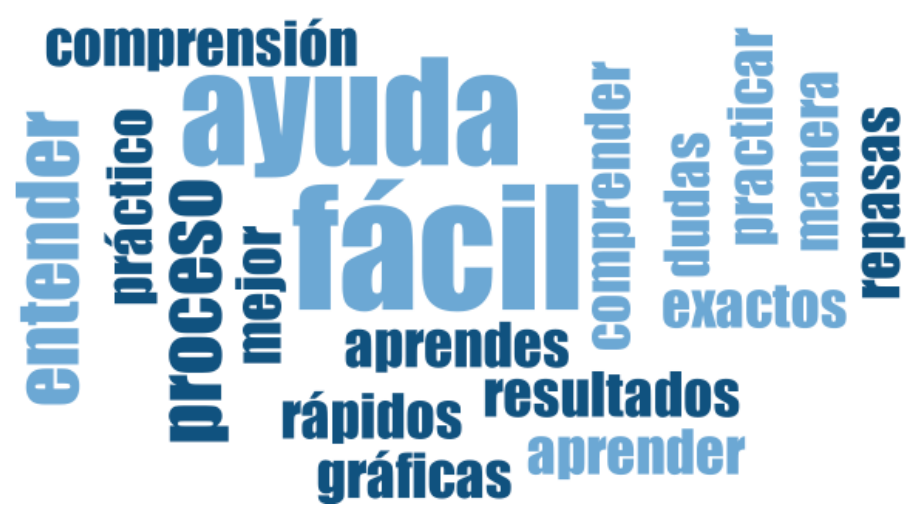

Figura 23. Nube de palabras para la variable Proceso de enseñanza-aprendizaje

Fuente: Elaboración propia

Asimismo, los universitarios están motivados de utilizar la tecnología en las actividades escolares debido a que la aplicación GeoGebra facilita el proceso educativo sobre la estadística:

- $\quad$ Sí, es muy fácil entender los temas (Estudiante 1, Masculino, 19 años, Comercio).

- $\quad$ Sí, te motiva porque lo puedes utilizar en cualquier lugar (Estudiante 16, Femenino, 19 años, Mercadotecnia).

- Sí, para aclarar dudas (Estudiante 33, Femenino, 20 años, Mercadotecnia).

De acuerdo con los participantes, los principales beneficios de la aplicación GeoGebra son Fácil, Aprendizaje, Rápido y Eficaz:

- Fácil, rápido y aprendizaje (Estudiante 4, Masculino, 18 años, Administración).

- Fácil, eficaz y práctico (Estudiante 25, Femenino, 20 años, Administración).

- $\quad$ Fácil y aprendizaje (Estudiante 26, Femenino, 19 años, Mercadotecnia).

Incluso, los alumnos están satisfechos de utilizar la aplicación GeoGebra en la asignatura Instrumentación estadística para los negocios debido a que esta herramienta tecnológica facilita el 
proceso de enseñanza-aprendizaje sobre la Probabilidad Binomial:

- Sí, ya que es de gran ayuda para estudiar (Estudiante 26, Femenino, 19 años, Mercadotecnia).

- Sí, me ayuda a entender el contenido (Estudiante 27, Femenino, 19 años, Mercadotecnia).

- Sí, me ayuda a repasar lo visto en clase (Estudiante 31, Masculino, 19 años, Comercio).

Las palabras más frecuentes sobre la variable Empleo de la aplicación GeoGebra son Fácil, Aprendizaje, Práctico, Dinámico y Eficaz (Ver Figura 24).

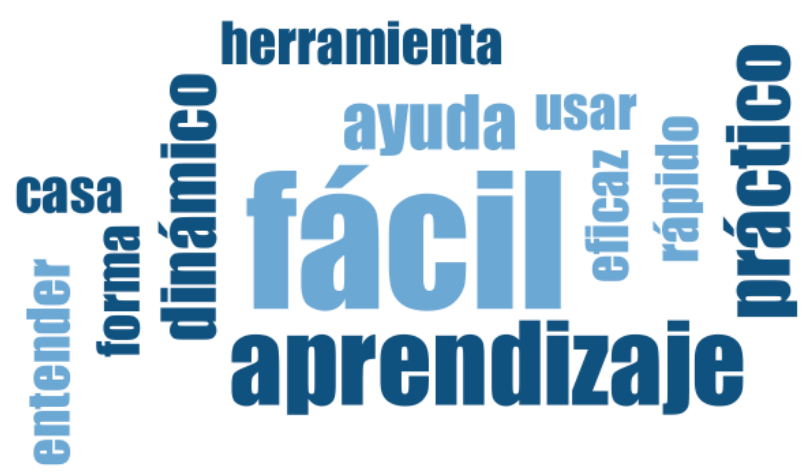

Figura 24. Nube de palabras para la variable Empleo de la aplicación GeoGebra

Fuente: Elaboración propia

Los encuestados mencionan que el diseño de la aplicación GeoGebra es visual, amigable y fácil de usar:

- Sí, porque es más visual (Estudiante 3, Masculino, 20 años, Comercio).

- $\quad$ Sí, es muy visual (Estudiante 9, Masculino, 18 años, Mercadotecnia).

- $\quad$ Sí, es muy amigable y fácil de usar (Estudiante 31, Masculino, 19 años, Comercio).

Además, los universitarios consideran que la aplicación GeoGebra es una herramienta innovadora para el campo educativo:

- Sí, bastante ya que con ella aprendes más (Estudiante 43, Masculino, 19 años, Mercadotecnia).

- Sí, es una herramienta dinámica e innovadora (Estudiante 49, Masculino, 21 años, Mercadotecnia).

- Sí, es otra forma de hacer las cosas (Estudiante 50, Femenino, 18 años, Contaduría).

Por último, los alumnos de la asignatura Instrumentación estadística para los negocios consideran que la aplicación GeoGebra es una herramienta web muy útil para el campo educativo:

- Sí, es más fácil comprender los temas (Estudiante 26, Femenino, 19 años, Mercadotecnia).

- Sí, debido a la interacción (Estudiante 33, Femenino, 20 años, Mercadotecnia).

- Sí, nos ayuda a reforzar lo visto en clase (Estudiante 38, Femenino, 19 años, Contaduría). 
Las palabras más frecuentes sobre la variable Usabilidad de la aplicación GeoGebra son Fácil, Herramienta, Usar, Apoyo, Ayuda e Innovadora (Ver Figura 25).

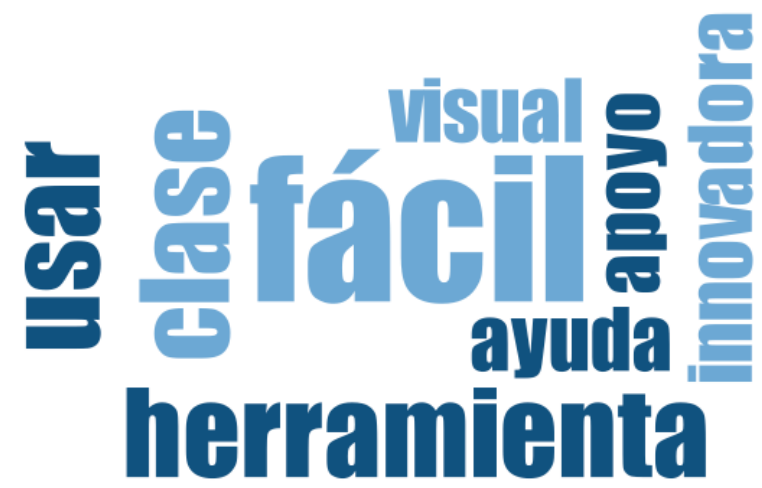

Figura 25. Nube de palabras para la variable Usabilidad de la aplicación GeoGebra

Fuente: Elaboración propia

\section{Discusión}

Esta investigación cualitativa y cuantitativa comparte las ideas de diversos autores (p.ej., Hobbs y Tuzel, 2017; Kramer, Neugebauer, Magenheim y Huppertz, 2015; Scott, Nerminathan, Alexander, Phelps y Harrison, 2017) sobre la necesidad de incorporar la tecnología en el campo educativo con el propósito de innovar las actividades escolares.

El uso de la tecnología en las actividades escolares permite la creación de espacios virtuales ideales para lograr el aprendizaje flexible y mejorar la calidad de la experiencia educativa (Agostinho, Lockyer y Bennett, 2018; Hu, Gong, Lai y Leung, 2018). De hecho, la incorporación de las herramientas digitales en las actividades escolares favorece el desarrollo de las habilidades en los estudiantes (Erdem, Uzal y Saka, 2018; Yang, Kuo, Ji y McTigue, 2018).

En particular, la aplicación GeoGebra mejora la asimilación del conocimiento, facilita el desarrollo de las habilidades matemáticas y propicia el rol activo en los estudiantes durante el proceso de enseñanza-aprendizaje sobre la Probabilidad Binomial. Los resultados sobre la regresión lineal obtenidos de la hoja de cálculo y el aprendizaje máquina con $60 \%, 70 \%$ y $80 \%$ de entrenamiento indican que los contenidos, la estética y la simulación de la aplicación GeoGebra influyen positivamente el proceso educativo sobre la estadística.

\section{a. Contenidos de la aplicación GeoGebra}

La mayoría de los estudiantes consideran que los contenidos de GeoGebra facilitan totalmente $(n=40,65.57 \%)$ el proceso de enseñanza-aprendizaje. Los resultados de la hipótesis 1 sobre el aprendizaje automático superan el valor de 0.749 , por consiguiente, los contenidos de la aplicación GeoGebra influye positivamente la asimilación del conocimiento sobre la Probabilidad Binomial.

Los resultados de la hipótesis 2 sobre el aprendizaje automático superan el valor de 0.337 , por lo tanto, los contenidos de la aplicación GeoGebra influye positivamente el desarrollo de las habilidades matemáticas sobre la Probabilidad Binomial. Asimismo, los resultados de la hipótesis 3 sobre el aprendizaje automático superan el valor de 0.425 , por consiguiente, los contenidos de la aplicación GeoGebra influye positivamente el rol activo del estudiante durante el aprendizaje de la 
Probabilidad Binomial.

La técnica árbol de decisión establece 3 modelos predictivos sobre los Contenidos de la aplicación GeoGebra y el proceso educativo (asimilación del conocimiento, desarrollo de las habilidades matemáticas y rol activo del estudiante) con una exactitud superior al $78.60 \%$.

\section{b. Estética de la aplicación GeoGebra}

La mayoría de los estudiantes consideran que la estética de GeoGebra facilita totalmente $(n=40$, $65.57 \%$ ) el proceso de enseñanza-aprendizaje. Los resultados de la hipótesis 4 sobre el aprendizaje automático superan el valor de 0.390, por lo tanto, la estética de la aplicación GeoGebra influye positivamente la asimilación del conocimiento sobre la Probabilidad Binomial.

Los resultados de la hipótesis 5 sobre el aprendizaje automático superan el valor de 0.185 , por consiguiente, la estética de la aplicación GeoGebra influye positivamente el desarrollo de las habilidades matemáticas sobre la Probabilidad Binomial. Asimismo, los resultados de la hipótesis 6 sobre el aprendizaje automático superan el valor 0.310 , por lo tanto, la estética de la aplicación GeoGebra influye positivamente el rol activo del estudiante durante el aprendizaje de la Probabilidad Binomial.

La técnica árbol de decisión establece 3 modelos predictivos sobre la estética de la aplicación GeoGebra y el proceso de enseñanza-aprendizaje (asimilación del conocimiento, desarrollo de las habilidades matemáticas y rol activo del estudiante) con una exactitud superior al $80.30 \%$.

\section{c. Simulación de la aplicación GeoGebra}

La mayoría de los estudiantes consideran que la simulación de GeoGebra facilita totalmente $(n=41$, $67.21 \%$ ) el proceso de enseñanza-aprendizaje. Los resultados de la hipótesis 7 sobre el aprendizaje automático superan el valor de 0.570 , por consiguiente, la simulación de la aplicación GeoGebra influye positivamente la asimilación del conocimiento sobre la Probabilidad Binomial.

Los resultados de la hipótesis 8 sobre el aprendizaje automático superan el valor de 0.410 , por lo tanto, la simulación de la aplicación GeoGebra influye positivamente el desarrollo de las habilidades matemáticas sobre la Probabilidad Binomial. Asimismo, los resultados de la hipótesis 9 sobre el aprendizaje automático superan el valor de 0.230 , por lo tanto, la simulación de la aplicación GeoGebra influye positivamente el rol activo del estudiante durante el aprendizaje de la Probabilidad Binomial. La técnica árbol de decisión establece 3 modelos predictivos sobre la simulación de la aplicación GeoGebra y el proceso de enseñanza-aprendizaje (asimilación del conocimiento, desarrollo de las habilidades matemáticas y rol activo del estudiante) con una exactitud superior al $77.00 \%$.

La minería de datos (técnica árbol de decisión) determinó las condiciones favorables para la asimilación del conocimiento, el desarrollo de las habilidades matemáticas y el rol activo del estudiante por medio del género, la carrera, la edad y las características de la aplicación GeoGebra.

\section{d. Percepciones de los estudiantes sobre la aplicación GeoGebra}

La aplicación GeoGebra facilita la comprensión de los temas sobre la Probabilidad Binomial y ayuda a eliminar las dudas. Para la variable Proceso de enseñanza-aprendizaje, las palabras más frecuentes son Fácil, Ayuda, Entender, Proceso, Aprender, Comprender y Dudas.

Asimismo, los alumnos de las licenciaturas en Administración, Contaduría, Comercio, Informática y Mercadotecnia están satisfechos y motivados de utilizar la aplicación GeoGebra. Para la variable 
Empleo de la aplicación GeoGebra, las palabras más frecuentes sobre son Fácil, Aprendizaje, Práctico, Dinámico y Eficaz.

El diseño de la aplicación GeoGebra es visual, amigable y fácil de usar. Para la variable Usabilidad de la aplicación GeoGebra, las palabras más frecuentes sobre Fácil, Herramienta, Usar, Apoyo, Ayuda e Innovadora.

Por último, las TIC están modificando el comportamiento, las actitudes y las funciones de los docentes y alumnos durante la planeación y realización de las actividades escolares (Hobbs y Tuzel, 2017; Weidlich y Bastiaens, 2018).

\section{Conclusiones}

GeoGebra es una aplicación web idónea para el proceso de enseñanza-aprendizaje sobre la Probabilidad Binomial. De hecho, los resultados de la regresión lineal (aprendizaje automático con $60 \%, 70 \%$ y $80 \%$ de entrenamiento) indican que los contenidos, la estética y la simulación de esta aplicación influyen positivamente la asimilación del conocimiento, el desarrollo de las habilidades matemáticas y el rol activo de los estudiantes.

La técnica árbol de decisión (ciencia de datos) permite identificar los condiciones favorables para el proceso de enseñanza-aprendizaje sobre la Probabilidad Binomial por medio de la aplicación GeoGebra.

Asimismo, el análisis cualitativo señala que los alumnos de la asignatura Instrumentación estadística para los negocios están motivados y satisfechos de la incorporación de la tecnología en el campo educativo.

Las limitaciones de este estudio están relacionadas con el uso de la aplicación GeoGebra para calcular la Probabilidad Binomial. Por consiguiente, las futuras investigaciones pueden analizar el impacto de esta aplicación en el proceso de enseñanza-aprendizaje sobre la Probabilidad de Pascal, Hipergeométrica y Poisson.

Asimismo, este estudio recomienda el uso de la aplicación GeoGebra en el campo educativo sobre la estadística debido a que esta herramienta web tiene una interfaz sencilla, agradable, útil y rápida.

Por último, los avances de las tecnologías están transformando las funciones de los docentes y alumnos en el contexto educativo. De hecho, los alumnos de las Licenciaturas en Administración, Comercio, Contaduría, Informática y Mercadotecnia adquirieron un rol activo durante la realización de las actividades escolares por medio de la aplicación GeoGebra.

\section{Referencias}

Agostinho, S., Lockyer, L. y Bennett, S. (2018). Identifying the characteristics of support Australian university teachers use in their design work: Implications for the learning design field. Australasian Journal of Educational Technology, 34(2), 1-15. doi: https://doi.org/10.14742/ajet.3776

Alkis, N. y Taskaya Temizel, T. (2018). The Impact of Motivation and Personality on Academic Performance in Online and Blended Learning Environments. Educational Technology \& Society, 21 (3), 35-47. 
Banerjee, G. y Murthy, S. (2018). CuVIS: An interactive tool for instructors to create effective customized learning designs with visualizations. Australasian Journal of Educational Technology, 34(2), 95-116. doi: https://doi.org/10.14742/ajet.3773

Bhagat, K. K. y Chang, C. Y. (2015). Incorporating GeoGebra into Geometry learning: a lesson from India. Eurasia Journal of Mathematics, Science \& Technology Education, 11(1), 77-86.

Dolan, E., Hancock, E. y Wareing, A. (2015). An evaluation of online learning to teach practical competencies in undergraduate health science students. The Internet and Higher Education, 24, 21-25. doi: https://doi.org/10.1016/j.iheduc.2014.09.003

Erdem, A., Uzal, G. y Saka, M. (2018). High School Students' Proficiency Perceptions to the Usage of Technology Products at Physics Lessons. The Turkish Online Journal of Educational Technology, 17(2), 20-32. Recuperado de http://www.tojet.net/articles/v17i2/1726.pdf

Granberg, C. y Olsson, J. (2015). ICT-supported problem solving and collaborative creative reasoning: Exploring linear functions using dynamic mathematics software. The Journal of Mathematical Behavior, 37, 48-62. doi: https://doi.org/10.1016/j.jmathb.2014.11.001

Hernández Guerrero, J. I., Acosta Gonzaga, E. A., Gordillo Mejía, A. y Sandoval Gómez, R. J. (2018). Sistema para la enseñanza de matemáticas basado en evaluación formativa. En F. Santillán Campos, Docencia e investigación, mecanismos de reflexión y cambio en Latinoamerica (pp. 24-38). Ciudad de México: Cenid.

Hobbs, R. y Tuzel, S. (2017). Teacher motivations. British Journal of Educational Technology, 48, 7-22. doi: https://doi.org/10.1111/bjet.12326

Hu, X., Gong, Y., Lai, C. y Leung, F. S. (2018). The relationship between ICT and student literacy in mathematics, reading, and science across 44 countries: A multilevel analysis. Computers \& Education, 125, 1-13. doi: https://doi.org/10.1016/j.compedu.2018.05.021

Jaffar, M. A. y Musa R. (2019). Factorial Validation and Measurement Model of Attitude and Intention Towards Adoption of Islamic Financing Among Non-users. En F. Hassan, I. Osman y E.S. Kassim (eds.), Contemporary Management and Science Issues in the Halal Industry (pp. 65-76). Estados Unidos: Springer.

Khalil, M., Farooq, R. A., Cakıroglu, E., Khalil, U. y Khan, D. M. (2018). The Development of Mathematical Achievement in Analytic Geometry of Grade-12 Students through GeoGebra Activities. Eurasia Journal of Mathematics, Science and Technology Education, 1-11. doi: https://doi.org/10.29333/ejmste/83681

Kramer, B. J., Neugebauer, J., Magenheim, J. y Huppertz, H. (2015). New ways of learning. British Journal of Educational Technology, 46, 965-971. doi: https://doi.org/10.1111/bjet.12301

Landa Alemán, A. A., Martínez Cortés, J., López Orozco, G. y Ramírez Ramírez, V. H. (2018). El uso de la plataforma educativa Edmodo como apoyo para el aprendizaje del francés como lengua extranjera en el nivel superior. En F. Santillán Campos, Docencia e investigación, mecanismos de reflexión y cambio en Latinoamerica (pp. 83-92). Ciudad de México: Cenid.

Mavroudi, A. y Tsagari, D. (2018). Profiling of English language teachers as trainees in an online course and ensuing implications. Computers \& Education, 126, 1-12. doi: https://doi.org/10.1016/j.compedu.2018.06.029

Porat, E., Blau, I. y Barak, A. (2018). Measuring digital literacies: Junior high-school students' perceived competencies versus actual performance. Computers \& Education, 126, 23-36. doi: https://doi.org/10.1016/j.compedu.2018.06.030

Rooij, S. W. y Zirkle, K. (2016). Balancing pedagogy, student readiness and accessibility: A case study in collaborative online course development. The Internet and Higher Education, 28, 1-7. doi: https://doi.org/10.1016/j.iheduc.2015.08.001

Salas Rueda, R. A. (2018). Uso del servicio en la nube GeoGebra durante el proceso enseñanza-aprendizaje sobre las matemáticas. Revista iberoamericana para la investigación y el desarrollo educativo, 8, 2352. Recuperado de https://www.ride.org.mx/index.php/RIDE/article/view/331 
Salas Rueda, R. A. y Salas Silis, J. A. (2018). Simulador Logic.ly ¿Herramienta tecnológica para facilitar el proceso enseñanza-aprendizaje sobre las Matemáticas? Revista Dilemas Contemporáneos: Educación, Política y Valores, 5(3), 1-25.

Salas Rueda, R. A. y Vázquez Estupiñán, J. J. (2017). Innovación en el proceso educativo superior a través del servicio en la nube erpag. Revista electrónica calidad en la educación superior, 8(2), 62-86. Recuperado de http://dx.doi.org/10.22458/caes.v8i2.1917

Salas Rueda, R. A., Vázquez Estupiñán, J. J. y Lugo García, J. L. (2016). Uso del avatar en el proceso de enseñanza aprendizaje sobre las aplicaciones de las derivadas. Revista de Comunicación de la SEECI, 39,71-84. doi: http://dx.doi.org/10.15198/seeci.2016.39.72-88

Scott, K. M., Nerminathan, A., Alexander, S., Phelps, M. y Harrison, A. (2017). Mobile devices for learning in clinical settings. British Journal of Educational Technology, 48, 176-190. doi: https://doi.org/10.1111/bjet.12352

Takaci, D., Stankov, G. y Milanovic, I. (2015). Efficiency of learning environment using GeoGebra when calculus contents are learned in collaborative groups. Computers \& Education, 82, 421-43. doi: https://doi.org/10.1016/j.compedu.2014.12.002

Wassie, Y. A. y Zergaw, G. A. (2019). Some of the Potential Affordances, Challenges and Limitations of Using GeoGebra in Mathematics Education. Eurasia Journal of Mathematics, Science and Technology Education, 15(8), 1-11. doi: https://doi.org/10.29333/ejmste/108436

Weidlich, J. y Bastiaens, T. (2018). Technology Matters - The Impact of Transactional Distance on Satisfaction in Online Distance Learning. International Review of Research in Open and Distributed Learning, 19(3), 222-242.

Yang, X., Kuo, L. J., Ji, X. y McTigue, E. (2018). A critical examination of the relationship among research, theory, and practice: Technology and reading instruction. Computers \& Education, 125, 62-73. doi: https://doi.org/10.1016/j.compedu.2018.03.009

Zulnaidi, H. y Zamri, S. N. (2017). The Effectiveness of the GeoGebra Software: The Intermediary Role of Procedural Knowledge On Students' Conceptual Knowledge and Their Achievement in Mathematics. Eurasia Journal of Mathematics Science and Technology Education, 13(6), 2155-2180. 